\title{
TITLE:
}

\section{Open algebraic surfaces with finite group actions}

$\operatorname{AUTHOR}(S)$ :

Miyanishi, M.; Masuda, K.

\section{CITATION:}

Miyanishi, M....[et al]. Open algebraic surfaces with finite group actions. 代数幾何学シンポ ジューム記録 2000, 2000: 203-225

\section{ISSUE DATE:}

2000

URL:

http://hdl.handle.net/2433/214714

RIGHT: 


\title{
Open algebraic surfaces with finite group actions
}

\author{
M. Miyanishi and K. Masuda
}

\section{Introduction}

Let $G$ be a finite group. Consider the set of $\log$ projective surfaces $(\bar{V}, \bar{\Delta})$ defined over a. fixed, algebraically closed, gound field of characteristic zero which admit effective algebraic $G$-actions. We say that a morphism $f:(\bar{V}, \bar{\Delta}) \rightarrow(\bar{W}, \bar{\Gamma})$ is a $G$-morphism (or G-equivariant morphism) if $f$ commutes with the $G$-actions. We can define the notion of relatively minimal (or minimal) model with respect to the birational $G$-morphisms.

The objective of the present article is to consider the equivariant classification of such $G$-relatively minimal $\log$ projective surfaces in the case where the log Kodaira dimension of $(\bar{V}, \bar{\Delta})$ is $-\infty$, i.e., $\bar{\kappa}(\bar{V}-\bar{\Delta} \cup \operatorname{Sing} \bar{V})=-\infty$. Our attempt is achieved under some technical hypotheses which enable us to make use of the Mori theory, but it still reveals some phenomena which are particular to the equivariant settings (cf. Theorems 1.5, 2.6).

We are motivated by Zhang [5] to consider this kind of equivariant classification. In the case $\bar{V}$ is a smooth rational surface and $\bar{\Delta}=0$, the results treated in the present article are, in fact, contained in [5].

\section{Preliminaries}

Let $(\bar{V}, \bar{\Delta})$ be a log projective surface (cf. [4]). Namely, $\bar{V}$ is a normal projective surface and $\bar{\Delta}$ is a reduced effective Weil divisor such that $(\bar{V}, \bar{\Delta})$ has $\log$ terminal singularities. Suppose that a finite group $G$ acts algebraically on $\bar{V}$ in such a way that $\bar{\Delta}$ is $G$-stable. Let $\bar{W}$ be the algebraic quotient $\bar{V} / / G$ and let $\pi: \bar{V} \rightarrow \bar{W}$ be the quotient morphism. Set $\bar{\Gamma}=\pi_{*}(\bar{\Delta})$. Then $\bar{W}$ is a normal projective surface with at worst quotient singularities. Let $f:(V, D) \rightarrow(\bar{V}, \bar{\Delta})$ be the minimal resolution and let $X=\bar{V}-\bar{\Delta} \cup \operatorname{Sing} \bar{V}$.

We assume that the following conditions hold:

(1) $(\bar{W}, \bar{\Gamma})$ ia a log projective surface. Hence the singularities of $\bar{W}$ lying on $\bar{\Gamma}$ are cyclic quotient singularities whose resolution graph has one (and only one) terminal component meeting the proper transform of $\bar{\Gamma}$ transversally in one point.

(2) $\bar{\kappa}(V-D)=-\infty$ and that $\left[D^{\#}\right]=\Delta$, where $\Delta$ is the proper transform of $\bar{\Delta}$ on $V$. The second condition implies that all possible rational admissible maximal twigs (resp. rods, forks) of $D$ are peeled off.

By applying the Mori theory to the quotient surface $\bar{W}$, we have

$$
\overline{\mathrm{NE}}(\bar{W})=\overline{\mathrm{NE}}_{\left(\bar{\Gamma}+K_{\bar{W}}\right) \geq 0}(\bar{W})+\sum_{\bar{F} \in \mathcal{F}^{\prime}} \mathbf{R}_{+} \bar{F},
$$


where $\mathcal{F}^{\prime}$ is a countable set of the extremal curves $\bar{F}$ on $\bar{W}$ with $\bar{F}$ being represented by an irreducible curve $\bar{C}$ on $\bar{V}$ and where

$$
\overline{\mathrm{NE}}_{\left(\bar{\Gamma}+K_{\bar{W}}\right) \geq 0}(\bar{W})=\left\{\eta \in \overline{\mathrm{NE}}(\bar{W}) \mid\left(\eta \cdot \bar{\Gamma}+K_{\bar{W}}\right) \geq 0\right\} .
$$

Since $\pi^{*}(\overline{\mathrm{NE}}(\bar{W}))=\overline{\mathrm{NE}}(\bar{V})^{G}$, we have

$$
\overline{\mathrm{NE}}(\bar{V})^{G}=\overline{\mathrm{NE}}_{\pi^{*}\left(\bar{\Gamma}+K_{\bar{W}}\right) \geq 0}(\bar{V})^{G}+\sum_{\bar{C} \in \mathcal{F}^{\prime}}\left(\sum_{g \in G} \bar{C}^{g}\right) \mathbf{R}_{+},
$$

where

$$
\overline{\mathrm{NE}}_{\pi^{*}\left(\bar{\Gamma}+K_{\bar{W}}\right) \geq 0}(\bar{V})^{G}=\left\{\xi \in \overline{\mathrm{NE}}(\bar{V})^{G} \mid\left(\xi \cdot \pi^{*}\left(\bar{\Gamma}+K_{\bar{W}}\right)\right) \geq 0\right\}
$$

Note that

$$
\bar{\Delta}+K_{\bar{V}}=\pi^{*}\left(\bar{\Gamma}+K_{\bar{W}}\right)+\bar{R}
$$

where $\bar{R}$ is the $\log$ ramification divisor which is an effective divisor (cf. litaka [2]). We assume, furthermore, that

(3) $\bar{R}$ is a nef divisor on $\bar{V}$.

Then it is clear that

$$
\begin{aligned}
\overline{\mathrm{NE}}_{\pi^{*}\left(\bar{\Gamma}+K_{\bar{W}}\right) \geq 0}(\bar{V})^{G} & \subseteq \overline{\mathrm{NE}}_{\left(\bar{\Delta}+K_{\bar{V}}\right) \geq 0}(\bar{V})^{G} \\
& :=\left\{\xi \in \overline{\mathrm{NE}}(\bar{V})^{G} \mid\left(\xi \cdot \bar{\Gamma}+K_{\bar{W}}\right) \geq 0\right\} .
\end{aligned}
$$

Hence we obtain the following result.

Lemma 1.1 With the above notations and assumptions, we have

$$
\overline{\mathrm{NE}}(\bar{V})^{G}=\overline{\mathrm{NE}}_{\left(\bar{\Delta}+K_{\bar{V}}\right) \geq 0}(\bar{V})^{G}+\sum_{\bar{C} \in \mathcal{F}}\left(\sum_{g \in G} \bar{C}^{g}\right) \mathbf{R}_{+},
$$

where $\mathcal{F}$ is a countable subset of $\mathcal{F}^{\prime}$.

Let $\bar{F}=\left(\sum_{g \in G} \bar{C}^{g}\right) /|H|$ be a $G$-invariant extremal curve, where $H$ is the isotropy group of an irreducible component $C$, i.e., $H=\left\{g \in G \mid \bar{C}^{g}=\bar{C}\right\}$. We first assume that $\rho(\bar{V} / / G) \geq 2$. The case $\rho(\bar{V} / / G)=1$ will be treated later. Then there exists a nef divisor $\bar{H}$ on $\bar{V}$ such that $(\bar{H} \cdot \bar{F})=0$. Hence $\left(\bar{F}^{2}\right) \leq 0$ by the Hodge index theorem.

CASE 1. Suppose $\left(\bar{F}^{2}\right)<0$. Since $\left(\bar{F} \cdot \bar{\Delta}+K_{\bar{V}}\right)<0$, we have $\left(F \cdot D^{\#}+K_{V}\right)<0$, where $F=\sum_{g \in G} C^{g} /|H|$ is the proper transform of $F$. Moreover $\left(F^{2}\right)<0$. Hence, for the component $C$ of $F$, it follows that $\left(C \cdot D^{\#}+K_{V}\right)<0$ and $C+$ Bk $D$ is negative definite, for $\left(C \cdot D^{\#}+K_{V}\right)=\left(C^{g} \cdot D^{\#}+K_{V}^{r}\right)$ for every $g \in G$. To go further, we need the following result.

Lemma 1.2 With the above notations and assumptions, the following assertions hold: 
(1) $D^{\#}+K_{V}^{\prime}=f^{*}\left(\bar{\Delta}+K_{\bar{V}}^{\prime}\right)$ and $\mathrm{Bk} D$ are $G$-stable, and $\operatorname{Sing}(\bar{V})$ is a $G$-stable set.

(2) If $\left(F^{2}\right)<0$ and $C$ is not $G$-stable, then $C \cap C^{g}=\emptyset$ whenever $C^{g} \neq C$ for $g \in G$.

Proof. The assertion (1) is clear. We shall prove the assertion (2). Let $H$ be the isotropy subgroup of $C$, and let $\left\{g_{1}, \ldots, g_{s}\right\}$ be a set of representatives of the right coset decomposition $G / H$. Let $g_{1}=e$ the identity and let $C_{i}=C^{g_{i}}$. Suppose $C \neq C^{g}$ and $\left(C \cdot C^{g}\right)>0$. If $C \not \subset \Delta$, then $C_{i}$ is a $(-1)$ curve. Furthermore, each $C_{i}$ has at least one $C_{j}$ such that $\left(C_{i} \cdot C_{j}\right)>0$. Hence we have

$$
\begin{aligned}
\left(F^{2}\right) & =\left(\left(C_{1}+\cdots+C_{s}\right)^{2}\right) \\
& =\sum_{i=1}^{s}\left(C_{i} \cdot C_{1}+\cdots+C_{s}\right) \\
& \geq(-1+1)+\cdots+(-1+1)=0,
\end{aligned}
$$

which is a contradiction. Suppose $C \subset \Delta$. Since

$$
0>\left(C \cdot D^{\#}+K_{V}\right) \geq\left(C \cdot C+K_{V}^{\prime}\right),
$$

it follows that $C \cong \mathrm{P}^{1}$ and $\left(C^{2}\right)<0$, for $F+\mathrm{Bk} D$ is negative definite. Suppose $\left(C^{2}\right) \leq-2$. Since $C^{g} \in \Delta$ for $g \in G$ and $0>\left(C \cdot D^{\#}+K_{V}\right)$, it follows that $\left(C \cdot C^{g}\right)=0$ or 1 and $\left(C \cdot C^{g}\right)=1$ possibly for only one translate $C^{g}$. Furthermore, we have

$$
\left(C \cdot D^{\#}-\sum_{i=1}^{s} C_{i}\right)<\left\{\begin{array}{lllll}
1 & \text { if } & C \cap C^{g} \neq \emptyset & \text { for some } & g \in G-\{\varepsilon\} \\
2 & \text { if } & C \cap C^{g}=\emptyset & \text { for every } & g \in G-\{e\} .
\end{array}\right.
$$

Let $B_{1}, \ldots, B_{t}$ be all the irreducible components of $B \mathrm{k} D$ such that $\left(C \cdot B_{\mathbf{i}}\right)>0$. Then we have

$$
\left.\sum_{i=1}^{t}\left(1-\frac{1}{b_{i}}\right)<1 \quad \text { (or } 2\right),
$$

where $b_{i}=-\left(B_{i}{ }^{2}\right)$. This implies that $t \leq 1$ in the case $C \cap C^{g} \neq \emptyset$ and $t \leq 3$ if $C \cap C^{g}=\emptyset$ for every $g \in G-\{e\}$. In the case $t \leq 1$ or in the case $C \cap C^{g}=\emptyset$ for every $g \in G-\{e\}$ and $t=2$, the connected component of $F+\mathrm{Bk} D$ containing $C$ is an admissible rational rod. This contradicts the hypothesis that $\Delta=\left[D^{\#}\right]$. Hence $C$ is a $(-1)$ curve. In the case $C \cap C^{g}=\emptyset$ for every $g \in G-\{e\}$ and $t=3, C$ has three twigs sprouting from it. Let $d_{1}, d_{2}, d_{3}$ be the (absolute value of the) discriminants of the intersection of the three twigs. Then the condition $\left(C \cdot D^{\#}-C\right)<2$ is stated as

$$
\sum_{i=1}^{3}\left(1-\frac{1}{d_{i}}\right)<2 .
$$

Hence $\left\{d_{1}, d_{2}, d_{3}\right\}$ is, up to permutations, one of the Platonic triplets. Hence the connected component of $D$ containing $C$ is an admissible rational fork. This contradicts again the condition $\Delta=\left[D^{\#}\right]$. Hence $C$ is a $(-1)$ curve. The rest of the proof is the same as in the previous case $C \not \subset \Delta$.

Q.E.D. 
By Lemma 1.2, we can contract $\left\{C^{g} \mid g \in G\right\}$ simultaneously without losing the $G$ action if $\left(\bar{F}^{2}\right)<0$. Since the condition $\left(\bar{F}^{2}\right)<0$ implies that $F+$ Bk $D$ is negative definite, this implies that the contraction of $\bar{F}$ on $\bar{V}$ produces again a log projective surface with a $G$ action (cf. [4]). We say that a $\log$ projective surface $(\bar{V}, \bar{\Delta})$ with an action of a finite group $G$ is $G$-relatively minimal if there is no curve $\bar{F}=\sum_{g \in G} \bar{C}^{g}$ such that $\left(\bar{F}^{2}\right)<0$ and $\left(\bar{F} \cdot \bar{\Delta}+K_{\bar{V}}\right)<0$. In terms of a minimal resolution $f:(V, D) \rightarrow(\bar{V}, \bar{\Delta})$, it is equivalent to saying that there is no curve $F=\sum_{g \in G} C^{g}$ on $V$ such that $\left(F^{2}\right)<0, C+B k D$ is negative definite and $\left(C \cdot D^{\#}+K_{V}\right)<0$. Given an extremal curve $\bar{F}=\sum_{g \in G} \bar{C}^{3}$, we may assume, without loss of generality, that $\left(\bar{F}^{2}\right)=0$, i.e., $F+B k D$ is not negative definite, but negative semi-definite.

CASE 2. Suppose $\left(\bar{F}^{2}\right)=0$. Write $F=\sum_{i=1}^{s} C_{i}$, which is the integral part of $f^{*}(\bar{F})$. Consider first the case $\left(F^{2}\right)<0$. We leave the case $\left(F^{2}\right)=0$ below. We then have the following result.

Lemma 1.3 Suppose that $\left(\vec{F}^{2}\right)=0$ and $\left(F^{2}\right)<0$. Then the following assertions hold.

(1) If $\left(\bar{C}^{2}\right)<0$, then the $C^{g}$ with $g \in G$ are the mutually disjoint $(-1)$ curves, and $C+\mathrm{Bk} D$ is negative definite, while $F+\mathrm{Bk} D$ is not negative definite. Furthermore, $C$ (hence $C^{g}$ as well) is not a component of $\Delta$.

(2) If $\left(\bar{C}^{2}\right)=0$, then $\left(\bar{C} \cdot \bar{C}^{g}\right)=0$ for every $g \in G$.

(3) There exists a $\mathbf{P}^{1}$-fibration $\bar{\rho}: \bar{V} \rightarrow B$ such that a multiple of $\bar{F}$ is a fiber of $\bar{\rho}$ and that $G$ preserves $\bar{\rho}$.

Proof. (1) If $\left(\bar{C}^{2}\right)<0$, then $C+\mathrm{Bk} D$ is negative definite. So, if $\left(F^{2}\right)<0$, the argument in CASE 1 works in this case as well. Hence the $C^{g}$ with $g \in G$ are the mutually disjoint $(-1)$ curves. We can contract the $C^{g}$ simultaneously, though some components of $\mathrm{Bk} D$ may not be contracted because $F+\mathrm{Bk} D$ is not negative definite. It is then clear that $F$ has two or more irreducible components. Suppose $C \subset \Delta$ (hence every $C^{g} \subset \Delta$ ). Since $(\bar{V}, \bar{\Delta})$ is a $\log$ projective surface, for each $g \in G$, the connected component of $F+\mathrm{Bk} D$ containing $C^{g}$ is an admissible rational rod of $\mathrm{Bk} D$ with one end component meeting $C^{g}$ in one point transversally. Then $F+\mathrm{Bk} D$ is negative definite, which is not the case. So, $C^{g}$ is not a component of $\Delta$.

(2) Since $\left(\bar{F}^{2}\right)=\left(\bar{C}^{2}\right)=0$, it is clear that $\left(\bar{C} \cdot \bar{C}^{g}\right)=0$ for every $g \in G$.

(3) If $\left(\bar{C}^{2}\right)<0$, then the assertion (1) shows that $f^{*}(\bar{F})$ consists of smooth rational curves. Suppose $\left(\bar{C}^{2}\right)=0$. Since $\left(\bar{C} \cdot \bar{\Delta}+K_{\bar{V}}\right)=\left(C \cdot D^{\#}+K_{V}\right)<0$, it follows that the proper transform $C=f^{\prime}(\bar{C})$ is a rational curve. Note that $V$ is a ruled surface because $\bar{\kappa}(X)=-\infty$. If the irregularity $q$ is positive, the curves in a connected component of $F+B k D$ are mapped to the same point by the Albanese morphism. Let $\bar{\rho}: \bar{V} \rightarrow B$ be the $\mathbf{P}^{1}$-fibration induced by the Albanese morphism of $V$. Then each connected component $\bar{L}$ of $\bar{F}$ is contained in a fiber of $\bar{\rho}$. Since $\left(\bar{F}^{2}\right)=0$, a multiple of $\bar{L}$ is a fiber of $\vec{\rho}$. Let $\ell$ be a general fiber of $\bar{\rho}$. Then $\ell$ is algebraically equivalent to $N \bar{L}$ with $N>0$. Hence $\ell^{g} \approx N \bar{L}^{g}$. Since $\left(\bar{L} \cdot \bar{L}^{g}\right)=0$ because $\bar{L}^{g}$ is also a connected component of $\bar{F}$, we have $\left(\ell \cdot \ell^{g}\right)=0$. This implies that $G$ preserves the fibration $\bar{\rho}$. 
Now suppose that $V$ is rational. Let $n$ be a positive integer. By the Riemann-Roch theorem, we have

$$
\begin{aligned}
h^{0}\left(n f^{*}(\bar{F})\right)-h^{1}\left(n f^{*}(\bar{F})\right) & =\frac{1}{2}\left(n f^{*}(\bar{F}) \cdot n f^{*}(\bar{F})-K_{V}\right)+1 \\
& =-\frac{n}{2}\left(f^{*}(\bar{F}) \cdot K_{V}\right)+1 \\
& >\frac{n}{2}\left(f^{*}(\bar{F}) \cdot D^{\#}\right)+1
\end{aligned}
$$

because

$$
\left(f^{*}(\bar{F}) \cdot D^{\#}+K_{V}\right)=\left(\bar{F} \cdot \bar{\Delta}+K_{\bar{V}}\right)<0 .
$$

Suppose $\left(f^{*}(\bar{F}) \cdot D^{\#}\right) \geq 0$. Then $h^{0}\left(n f^{*}(\bar{F})\right)>1$ for $n \geq 1$. So, $\left|n\left(f^{*}(\bar{F})\right)\right|$ defines a morphism $\rho: V \rightarrow B$ whose general fibers are irreducible. Since $\left(f^{*}(\bar{F}) \cdot K_{V}\right)<-\left(f^{*}(\bar{F})\right.$. $\left.D^{\#}\right) \leq 0$, it follows that $\rho$ is a $\mathbf{P}^{1}$-fibration. Furthermore, $\rho$ induces a $\mathbf{P}^{1}$-fibration $\bar{\rho}: \bar{V} \rightarrow B$ such that $\rho=\tilde{\rho} \cdot f$ and that a multiple of $f^{*}(\bar{C})$ is a fiber. By the same argument as above, we know that $G$ preserves the fibration $\bar{\rho}$. Suppose that $\left(f^{*}(\bar{F}) \cdot D^{\#}\right)<0$. Then $(\bar{F} \cdot \bar{\Delta})<0$. Hence each connected component $\bar{L}$ of $\bar{F}$ is a component of $\bar{\Delta}$ with $\left(\bar{L}^{2}\right)<0$. This case does not occur by the hypothesis $\left(\bar{F}^{2}\right)=0$.

Q.E.D.

The reducible fiber of the $\mathbf{P}^{1}$-fibration in Lemma 1.3 which is supported by a connected component of $\bar{F}$ can be specified as follows.

Lemma 1.4 Let $\Phi$ be a reduced, reducible fiber of the $\mathbf{P}^{1}$-fibration $\bar{\rho}: \bar{V} \rightarrow B$ in Lemma 1.3 whose support is a connected component $\bar{L}$ of $\bar{F}$. Write the proper transform $L:=f^{\prime}(\bar{L})$ on the minimal resolution $V$ of $\bar{V}$ as $L=\sum_{i=1}^{s} C_{i}$. Then every $C_{i}$ is a $(-1)$ curve. Let $G_{0}$ be the subgroup of $G$ consisting of elements $g$ with $\bar{F}^{g}=\bar{F}$. Furthermore, if $G$ is an abelian group and $G$ acts on $\Phi$ effectively, the possible configurations of $f^{*}(\Phi)$ are exhausted by the following list:

(1) $s=2, G_{0} \cong \mathbf{Z} / 2 \mathbf{Z}$ and the dual graph of $f^{*}(\Phi)$ is a linear graph which is the $(-2)$ chain (corresponding to the exceptional graph of a rational double point of type $A_{n}$ ) with two $(-1)$ curves meeting the both end components of the $(-2)$ chain.

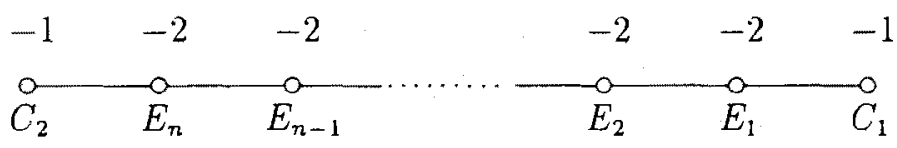

(2) $s=4, G_{0} \cong \mathbf{Z} / 2 \mathbf{Z} \times \mathbf{Z} / 2 \mathbf{Z}$, and the dual graph of $f^{*}(\Phi)$ is a linear graph which is the $(-2)$ chain (corresponding to the exceptional graph of a rational double point of type $\left.A_{n}\right)$ with two $(-1)$ curves meeting each of the both end components of the $(-2)$ chain. 


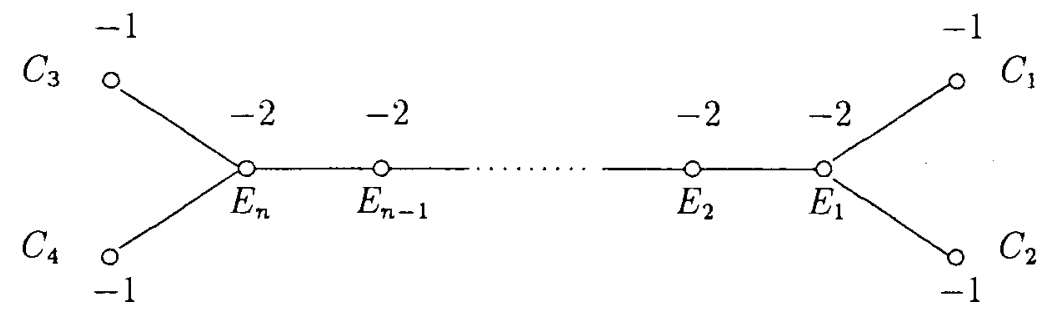

(3) $s=3, G_{0} \cong \mathbf{Z} / 3 \mathbf{Z}$ and the dual graph of $f^{*}(\Phi)$ is a (-3) component meeting three linear branches, each of which consists of a $(-2)$ curve and $(-1)$ curve with the $(-2)$ curve meeting the $(-3)$ component.

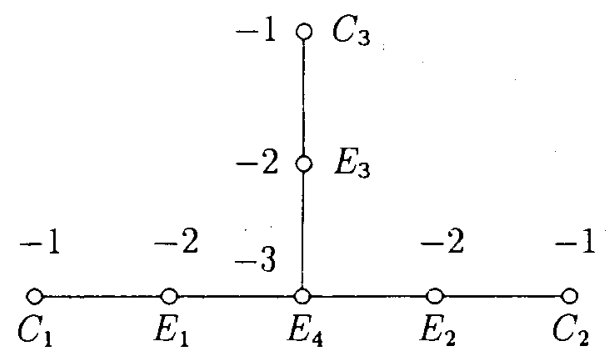

(4) $s \geq 3$, the configuration of $f^{*}(\Phi)$ is a unique $(-s)$ curve $E$ meeting $s$ curves $C_{1}, \ldots, C_{s}$ which are $(-1)$ curves, and $G_{0}$ is a finite subgroup of $\mathrm{PGL}(2, k)$ acting transitively on the set $\left\{P_{1}, \ldots, P_{s}\right\}$ of $\mathbf{P}^{1}$ with $P_{i}=C_{i} \cap E$.

In particular, the fiber $\Phi$ has a unique singular point.

Proof. Note that $f^{*}(\Phi)$ is a degenerate fiber of a $\mathbf{P}^{1}$-fibration on a smooth projective surface. Write

$$
f^{*}(\Phi)=\sum_{i=1}^{s} a C_{i}+\sum_{j=1}^{n} b_{j} E_{j},
$$

where the coefficients of the $C_{i}$ are the same becasuse every $C_{i}$ is a translate of one of the $C_{i}$ and where the $B_{j}$ are the exceptional curves of the minimal resolution $f: V \rightarrow \bar{V}$. Here $s \geq 2$ and $a=1$ because $\Phi$ is a reduced and reducible fiber by the hypothesis. Note that every $C_{i}$ is an end component of $f^{*}(\Phi)$. This implies that every $\bar{C}_{i}:=f\left(C_{i}\right)$ passes through one and only one singular point on $\Phi$. Since this singular point is a quotient singular point, the exceptional graph is either a rod or a fork.

Suppose first that the exceptional graph $E:=\sum_{j=1}^{n} E_{j}$ is a rod with $n \geq 2$. So, we may assume that $\left(E_{i} \cdot E_{j}\right)=1$ (resp. 0$)$ if $j=i+1$ with $1 \leq i<n$ (resp. otherwise). Hence $E_{1}$ and $E_{n}$ are the end components. Note that $C_{i}$ meets $E_{1}$ or $E_{n}$, for otherwise the coefficient $a$ mut be larger than 1 . Suppose $C:=C_{1}$ meets $E_{1}$. We claim that the set $H$ of elements $g \in G_{0}$ with $\left(C^{g} \cdot E_{1}\right)=1$ is a subgroup of $G_{0}$. In fact, if $g, h \in H$, then $\left(C^{g} \cdot E_{1}\right)=\left(C^{h} \cdot E_{1}\right)=1$. Suppose $\left(C^{g h} \cdot E_{n}\right)=1$. Then $h$ maps the point $C^{g} \cap E_{1}$ onto the point $C^{g h} \cap E_{n}$ on $E_{n}$. Hence $h$ maps $E_{1}$ onto $E_{n}$. So, $\left(C^{h} \cdot E_{1}\right)=0$. This is a contradiction. 
Indeed, $H$ is a normal subgroup of $G_{0}$ of index 2 . In particular, $s$ is even. If $s=2$, then the dual graph of $f^{*}(\Phi)$ is the case (1) listed above. So, assume that $s \geq 4$. We cliam that the stabilizer group of $C$ is trivial. In fact, let $K=\left\{g \in H \mid C^{g}=C\right\}$. Then the stabilizer group of $C_{i}$ is a conjugate of $K$. Hence $K$ acts on the curve $E_{1}$ and fixes at least three points $C_{1} \cap E_{1}, C_{i} \cap E_{1}$ and $E_{2} \cap E_{1}$, where $\left(C_{i} \cdot E_{1}\right)=1$. So, $K$ acts trivially on $E_{1}$. Consider the quotient space $V_{1}:=V / / K$. Since $G$ preserves the $\mathbf{P}^{1}$-fibration $\rho: V \rightarrow B$, there exists a $\mathbf{P}^{1}$-fibration $\rho_{1}: V_{1} \rightarrow B_{1}$. Then $C=\pi_{1}^{*}\left(\pi_{1}(C)\right)$, where $\pi_{1}: V \rightarrow V_{1}$ is the quotient morphism. Hence $\left(C^{2}\right)=\left|K^{2}\right|\left(\pi_{1}(C)^{2}\right)$. Since $C$ is a $(-1)$ curve, it follows that $|K|=1$. Now the group $H$ acts on $E_{1}$ so that the point $E_{1} \cap E_{2}$ is a fixed point. Sirice $H$ acts effectively on the affine line $E_{1}-E_{1} \cap E_{2}, H$ is a cyclic group of order $t$, where $s=2 t$. We assume that $C_{i} \cap E_{1} \neq \emptyset$ for $1 \leq i \leq t$ and $C_{i} \cap E_{n} \neq \emptyset$ for $t+1 \leq i \leq s$. After the contraction of $C_{1}, \ldots, C_{t}$, the component $E_{1}$ becomes a $(-1)$ curve. Hence $\left(E_{1}{ }^{2}\right)=-(t+1)$. Set $P:=E_{1} \cap E_{2}$. The action of $H$ near the point $P$ is given as $(x, y) \mapsto\left(\zeta x, \zeta^{d} y\right)$, where $\zeta$ is a primitive $t$-th root of unity, $\{x, y\}$ is a system of local parameters at $P$ such that $E_{1}$ (resp. $E_{2}$ ) is defined by $y=0$ (resp. $x=0$ ) and $d$ is an integer $0 \leq d<t$. Consider the quotient space $V_{2}:=V / / H$ with quotient morphism $\pi_{2}: V \rightarrow V_{2}$. Let $\bar{E}_{1}$ and $\bar{E}_{2}$ be the images of $E_{1}$ and $E_{2}$ by $\pi_{2}$, respectively. The point $\bar{P}:=\pi_{2}(P)$ is a cyclic quotient singular point of type $(t, d)$ if $d>0$ and a smooth point if $d=0$. By Sublemma below, we have $\left(\bar{E}_{1}{ }^{2}\right)=\left(E_{1}^{\prime 2}\right)+(d / t)$, where $E_{1}^{\prime}$ is the proper transform of $\bar{E}_{1}$ on the minimal resolution of $V_{2}$. We then have

$$
-(t+1)=\left(E_{1}^{2}\right)=t\left(\bar{E}_{1}^{2}\right)=t\left(\left(E_{1}^{\prime 2}\right)+\frac{d}{t}\right) .
$$

Since $\left(E_{1}^{\prime 2}\right)$ is an integer, it follows that $d=t-1$ and $\left(E_{1}^{\prime 2}\right)=-2$. Then we can cntract $H$-equivariantly the components $C_{1}, \ldots, C_{t}$ and $E_{1}$. On the minimal resolution of $V_{2}$, we contract the component $E_{1}^{\prime}$ and the linear chain of length $t-1$ of the exceptional $(-2)$ curves arising from the resolution of singularity of the point $\bar{P}$. Repeating the above argument, we find that the configuration of the curves $\sum_{i=1}^{s} C_{i}+\sum_{j=1}^{n} E_{j}$ together with the $H$-actions are described as follows, where $P_{i}:=E_{i} \cap E_{i+1}$ for $1 \leq i<n$ and $\zeta$ is a primitive $t$-th root of unity. Furthermore, we let $\left\{x_{i}, y_{i}\right\}$ be a system of local parameters such that $E_{i}$ (resp. $\left.E_{i+1}\right)$ is defined by $y_{i}=0$ (resp. $\left.x_{i}=0\right)$. The $H$-action near the point $P_{i}$ is given by $\left(x_{i}, y_{i}\right) \mapsto\left(\zeta x_{i}, \zeta^{-1} y_{i}\right)$.

Sublemma With the above notations, we have

$$
\left(\bar{E}_{1}^{2}\right)=\left({E_{1}^{\prime}}^{2}\right)+\frac{d}{t}
$$

Proof. Write $t / d$ in the form of a continued fraction

$$
\frac{t}{d}=a_{1}-\frac{1}{a_{2}-\frac{1}{\ddots}-\frac{1}{a_{s}}}
$$


Then the total transform of $\bar{E}_{1}+\bar{E}_{2}$ in the minimal resolution of singularity at $\bar{P}$ is a linear chain

$$
E_{1}^{\prime}+G_{1}+\cdots+G_{s}+E_{2}^{\prime}
$$

where $\left(G_{i}{ }^{2}\right)=-a_{i}$ for $1 \leq i \leq s$. Then the total transform of $\bar{E}_{1}$ in the minimal resolution is written as

$$
E_{1}^{\prime}+\alpha_{1} G_{1}+\alpha_{2} G_{2}+\cdots+\alpha_{s} G_{s}
$$

where $\alpha_{1}, \ldots, \alpha_{s}$ are determined by the conditions

$$
\begin{aligned}
& 1-a_{1} \alpha_{1}+\alpha_{2}=0 \\
& \alpha_{1}-a_{2} \alpha_{2}+\alpha_{3}=0 \\
& \\
& \alpha_{s-2}-a_{s-1} \alpha_{s-1}+\alpha_{s}=0 \\
& \alpha_{s-1}-a_{s} \alpha_{s}=0
\end{aligned}
$$

Then it is easy to verify that

$$
\left(\bar{E}_{1}^{2}\right)=\left(E_{1}^{\prime 2}\right)+\alpha_{1}=\left(E_{1}^{\prime 2}\right)+\frac{d}{t} .
$$

Q.E.D.

CASE $n=2 m+1$. The $H$-action stabilizes each of the components $E_{j}(1 \leq j \leq n)$, and $G_{0} / H$ flips the two branches $\sum_{i=1}^{t} C_{i}+\sum_{j=1}^{m} E_{j}$ and $\sum_{i=t+1}^{s} C_{i}+\sum_{j=m+2}^{n} E_{j}$. Let $\sigma$ be an element not in $H$. Then $\sigma\left(E_{m}\right)=E_{m+2}$. Since $\left|G_{0} / H\right|=2, \sigma^{2} \in H$. So, write $\sigma^{2}=\tau^{r}$, where $\tau \in H$ is a generator of $H$ and $0 \leq r<t$. Since $\sigma$ is an automorphism of finite order of $E_{m+1} \cong \mathbf{P}^{1}$, it fixes two points, say $Q_{1}, Q_{2}$, and acts on $\mathbf{A}_{*}^{1}:=\mathbf{P}^{1}-\left\{Q_{1}, Q_{2}\right\}$ as an element of $G_{m}$. So, if $r>0$, then the points $Q_{1}, Q_{2}$ coincide $P_{m}, P_{m+1}$ up to a permutation. This is a contradiction. Hence $\sigma^{2}=1$, and $\left\{Q_{1}, Q_{2}\right\} \cap\left\{P_{m}, P_{m+1}\right\}=\emptyset$. Note that $\sigma$ is an involution of the component $E_{m+1}$ commuting with the $H$-action. Write $E_{m+1}-\left\{P_{m}, P_{m+1}\right\}=\operatorname{Spec} k\left[x, x^{-1}\right]$, where $P_{m}$ and $P_{m+1}$ are respectively defined by $x=0$ and $x=\infty$. Choose the coordinate $x$ such that $Q_{1}$ is defined by $x=1$ and choose, furthermore, a generator $\tau$ of $H$ such that $\tau(x)=\zeta x$. Since $\sigma$ acts on an automorphism of $k\left[x, x^{-1}\right]$, we have $\sigma(x)=\alpha x^{-1}$ with $\alpha \in k^{*}$ because $\sigma\left(P_{m}\right)=P_{m+1}$. Since $\sigma\left(Q_{1}\right)=Q_{1}$ and $Q_{1}$ is defined by $x=1$, it follows that $\alpha=1$. So, $\sigma(x)=x^{-1}$. Then it is easy to show that $\sigma \tau \sigma=\tau^{-1}$. Thus $G_{0}$ is a dihedral group of order $2 t$, which is not abelian unless $t=2$. If $t=2$, then the graph of $\Phi$ is the case (2).

CASE $n=2 m$. Let $\sigma$ be an element of $G_{0}$ not in $H$. Then $G_{0}$ is generated by $\sigma$ and $H=\langle\tau\rangle$. Note that $H$ fixes the points $P_{i}(1 \leq i \leq n-1)$ and $\sigma$ fixes the mid-point $P_{m}$. Then $\sum_{i=1}^{s} C_{i}+\sum_{j \neq m, m+1} E_{j}$ is stabilized by $G_{0}$. Hence we can contract this divisor $G$-equivariantly. After the contraction, we obtain a smooth projective surface $V_{3}$ with a $\mathrm{P}^{\mathrm{l}}$-fibration $\rho_{3}: V_{3} \rightarrow B$ on which the group $G$ preserves the fibration $\rho_{3}$, i.e., $\rho_{3}$ maps the fibers of $\rho_{3}$ onto the fibers of $\rho_{3}$. The image of the fiber $\Phi$ consists of two $(-1)$ curves $\bar{E}_{m}$ and $\bar{E}_{m+1}$ meeting transversally in the point $\bar{P}_{m}$. The element $\sigma$ acts on the fiber in such a way that $\sigma\left(\bar{E}_{m}\right)=\bar{E}_{m+1}$. Then, by Theorem 2.6 below whose proof is independent of the present argument, $\sigma$ is an involution. Tracing the images of a point $P$ chosen on $E_{m}-\left\{P_{m}, P_{m-1}\right\}$ 
by the automorphisms $\sigma$ and $\tau$, we know that $\sigma \tau \sigma=\tau^{-1}$. So, we obtain the same result as in the case $n=2 m+1$.

Suppose next that the exceptional graph $E$ is a unique irreducible component. Then the curve $C_{i}$ intersects $E$ transversally in a point $P_{i}$. The restriction on the group $G$ is that $G$ is a finite subgroup of $\mathrm{PGL}(2, k)$ acting on the set $\left\{P_{1}, \ldots, P_{s}\right\}$ transitively. Hence the graph of $\Phi$ is the case (4).

Finally, consider the case the exceptional graph $E$ is a fork. Since a fork has a $(-2)$ component meeting the central component $E_{4}$, we denote the $(-2)$ component by $E_{1}$. There is only one $(-1)$ component $C_{1}$ meeting $E_{1}$. In fact, there is at most one $(-1)$ component meeting $E_{1}$. If there are none of them, then $E_{1}$ must remain after the contraction of all the $(-1)$ components and all subsequently contractible components of $f^{*}(\Phi)$. But this is not the case. On the other hand, if $g \in G$ maps $C_{1}$ to $C_{i}$, then $g$ maps $E_{1}$ to a $(-2)$ component of the exceptional graph which is adjacent to $C_{i}$. This observation shows that the curve $C_{i}$ and a $(-2)$ curve $E_{i}$ form a linear branch connected to the component $E_{4}$. The graph of $f^{*}(\Phi)$ is thus the case $(3)$ in the list. The group $G$ acts on the component $E_{4}$ and permutes three points $P_{i}:=E_{i} \cap E_{4}$ for $i=1,2,3$. Suppose that $G_{0}$ contains an element $\tau$ which permutes cyclically three points $P_{1}, P_{2}, P_{3}$. Then $G_{0} \cong \mathbf{Z} / 3 \mathbf{Z}$ as long as $G_{0}$ is abelian. Suppose that any element of $G_{0}$ fixes at least one point of $P_{1}, P_{2}, P_{3}$. Then there exist $\sigma_{1}, \sigma_{2}$ such that $\sigma_{1}\left(P_{1}\right)=P_{2}, \sigma_{2}\left(P_{1}\right)=P_{3}$. Then $\sigma_{1} \sigma_{2}$ permutes three points cyclically. This is a contradiction. Hence $G_{0} \cong \mathrm{Z} / 3 \mathrm{Z}$.

Q.E.D.

The observation in CASE $n=2 m+1$ in the proof of Lemma 1.4 implies the existence of a fiber $\Phi$ such that $f^{*}(\Phi)$ is a linear chain of $(-2)$ curves with $t$ of the (-1) curves meeting each of the end components of the chain if one admits the dihedral group. Here is an example.

EXAMPLE 1.5 Let $p: W \rightarrow B$ be a $\mathbf{P}^{1}$-fibration on a $\log$ projective surface $W$. We assume that the following conditions are satisfied.

(1) Let $H$ be a cyclic group of order $t$. The group $H$ acts on $W$ so that $\rho \cdot g=\rho$ for every $g \in G$. Hence, for every smooth fiber $F$ of $\rho$, there are two points $P_{0}, Q_{0}$ such that $P_{0}, Q_{0}$ are the fixed points and $H$ acts on $\mathbf{A}_{*}^{1}=F=\left\{P_{0}, Q_{0}\right\}$ via the natural $G_{m}$-action.

(2) Choose a smooth fiber $F$ as above. Choose an inhomogeneous coordinate $x$ on $F$ such that $P_{0}, Q_{0}$ are defined respectively by $x=0, x=\infty$ and that $\tau(x)=\zeta x$ for a generator $\tau$ of $H$, where $\zeta$ is a primitive $t$-th root of unity. Consider an involution $\sigma$ on $W$ such that $\sigma$ acts on $F$ as $\sigma(x)=x^{-1}$. Then $\sigma \tau \sigma=\tau^{-1}$ on $F$. Hence the subgroup $G$ of Aut $(W)$ generated by $\tau$ and $\sigma$ is the dihedral group of order $2 t$.

Such an example of $(W, p)$ with a G-action does exist. For example, consider the above $G$-action on $\mathbf{P}^{1}$ and take a direct product $\mathbf{P}^{1} \times B$.

Blow up the points $P_{0}, Q_{0}$ and obtain the exceptional curves $L_{1}, R_{1}$, respectively. By the abuse of notations, we denote the proper transform of $F$ by the same letter $F$ and denote the intersection points $F \cap L_{1}$ and $F \cap R_{1}$ by the letters $P_{0}$ and $Q_{0}$, respectively. Then the group $H$ acts on $L_{1}, R_{1}$. Near the point $P_{0}$ on $R_{1}$, choose a system of local parameters $\{x, y\}$ such that $x$ is as above and $y$ is an inhomogeneous coodinate on $L_{1}$ with the point $P_{0}$ defined by 
$y=0$. Then the action of $H$ on $L_{1}$ is given by $r(y)=\zeta^{-1} y$. Hence there is another $H$-fixed point $P_{1}$ on $L_{1}$. Similarly, there is a $H$-fixed point $Q_{1}$ on $R_{1}$. Blwo up the points $P_{1}, Q_{1}$ to obtain the exceptional curves $L_{2}, R_{2}$, respectively. Continue this process to obtain a linear chain

$$
L_{m}+L_{m-1}+\cdots+L_{1}+F+R_{1}+\cdots+R_{m-1}+R_{m},
$$

where we can extend the $G$-action onto the blown-up surfaces under which $\sigma\left(L_{i}\right)=R_{i}$ and $\sigma\left(R_{i}\right)=L_{i}$ for $1 \leq i \leq m$. Choose the points $A_{1}, \ldots, A_{t}$ on $L_{m}$ which constitute the $H$ orbit of $A_{1}$. Let $B_{j}=\sigma\left(A_{j}\right)$ for $1 \leq j \leq t$. Now blow up these $2 t$ points $A_{1}, \ldots, A_{t}$ and $B_{1}, \ldots, B_{t}$ to obtain the exceptional curves $C_{t+1}, \ldots, C_{s}$ and $C_{1}, \ldots, C_{t}$, respectively. Let $V$ be the surface obtained by the above sequence of blowing-ups. Then the dihedral group $G$ acts transitively on the set of $(-1)$ curves $\left\{C_{1}, \ldots, C_{t}, C_{t+1}, \ldots, C_{s}\right\}$. The surface $V$ has a $\mathbf{P}^{1}$ fibration $\rho: V \rightarrow B$ which extends the fibration $p$ and contains $\sum_{i=1}^{s} C_{i}+\sum_{j=1}^{m}\left(L_{j}+R_{j}\right)+F$ as a fiber. The linear chain $\sum_{j=1}^{m} L_{j}+F+\sum_{j+1}^{m} R_{j}$ which consists of $(-2)$ linear chains with two $-(t+1)$ curves attached to the end components contracts to a cyclic quotient singular point.

CASE 3. Now suppose that $\left(\bar{F}^{2}\right)=\left(F^{2}\right)=0$. It follows that $\bar{F} \cap \operatorname{Sing} \bar{V}=\emptyset$. Then we have the following result.

Lemma 1.6 Suppose $s \geq 2$, i.e., $C=C_{1}$ is not $G$-stable. Then the following assertions hold.

(1) Suppose $\left(C_{1} \cdot C_{i}\right) \neq 0$ for some $i \neq 1$. Then $F$ is rewritten as

$$
F=\sum_{i=1}^{r}\left(C_{i}+C_{i}^{\prime}\right),
$$

where $s=2 r,\left(C_{i}^{2}\right)=\left(C_{i}^{\prime 2}\right)=-1$ and $\left(C_{i} \cdot C_{i}^{\prime}\right)=1$. Hence there exists a $\mathbf{P}^{\mathbf{1}}$-fibration $\rho: V \rightarrow B$ such that the $G$-action preserves the $\mathbf{P}^{1}$ - fibration.

(2) Suppose $\left(C_{1} \cdot C_{i}\right)=0$ for every $i \neq 1$. Then $\left(C_{i}{ }^{2}\right)=0$ for every $i$, and there exists $a$ $\mathbf{P}^{1}$-fibration $\rho: V \rightarrow B$ such that the $G$-action preserves the $\mathbf{P}^{1}$-fibration.

(3) The $\mathbf{P}^{1}$-fibration $\rho: V \rightarrow B$ in (1) and (2) above factors as $\rho=\bar{\rho} \cdot f$, where $\bar{\rho}: \bar{V} \rightarrow B$ is a $\mathbf{P}^{\mathbf{I}}$-fibration.

Proof. (1) Since $\left(C_{i}{ }^{2}\right)<0$ and $\left(C_{i} \cdot D^{\#}+K_{V}\right)<0$, it follows that every $C_{i}$ is a $(-1)$ curve if $C_{i}$ is not contained in $\Delta$ for some $i$ and a smooth rational curve if $C_{i}$ is contained in $\Delta$. Set $\left(C_{i}{ }^{2}\right)=-a$ with $a \geq 1$ and $t=\left(C_{1} \cdot \sum_{i=2}^{s} C_{i}\right)$. Since every $C_{j}$ is a translate of $C_{1}$ by an element of $G$, we have $\left(C_{j} \cdot \sum_{i \neq j} C_{i}\right)=t$. Since $\left(F^{2}\right)=0$, we have

$$
\begin{aligned}
\left(F^{2}\right) & =\sum_{i=1}^{s}\left(C_{i} \cdot \sum_{j=1}^{s} C_{j}\right) \\
& =\sum_{i=1}^{s}\left\{\left(C_{i}^{2}\right)+\left(C_{i} \cdot \sum_{j \neq i} C_{j}\right)\right\} \\
& =-s a+s t,
\end{aligned}
$$


which yields that $t=a$ because $\left(F^{2}\right)=0$. We shall show that $a=1$, i.e., every $C_{i}$ is a $(-1)$ curve. Suppose $a \geq 2$. Since $\bar{\kappa}(X)=-\infty$, we know that $\left|D+K_{V}\right|=\emptyset$. Suppose that $V$ is rational. It then follows that $D$ and hence $\Delta$ has a tree as the dual graph. Since $C_{1}+\cdots+C_{s}$ is a part of $\Delta$, its dual graph is a tree. So, some component $C_{i}$ meets at most one component of $\sum_{j \neq i} C_{j}$. But the above remark shows that the number of the components in $\sum_{j \neq i} C_{j}$ that $C_{i}$ meets is exactly $a \geq 2$. This is a contradiction. If $V$ is irrational, $V$ is a ruled surface because $\left|n\left(D+K_{V}\right)\right|=\emptyset$ for every $n>0$. Since $C_{i}$ is a rational curve, it is a fiber component. Since the dual graph of a degenerate fiber of a $\mathbf{P}^{1}$-fibration has a tree as the dual graph, the dual graph of $C_{1}+\cdots+C_{s}$ is a tree. The above argument for the rational case works in the irrational case as well. Hence every $C_{i}$ is a $(-1)$ curve. Hence we may rewrite $F$ as

$$
F=\sum_{i=1}^{r}\left(C_{i}+C_{i}^{\prime}\right),
$$

where $\left(C_{i}{ }^{2}\right)=\left(C_{i}^{\prime 2}\right)=-1$ and $\left(C_{i} \cdot C_{i}^{\prime}\right)=1$. Since $\left(\left(C_{i}+C_{i}^{\prime}\right)^{2}\right)=0$, a complete linear system $\Lambda=\left|N\left(C_{1}+C_{1}^{\prime}\right)\right|$ with $N \gg 0$ defines a $\mathbf{P}^{1}$-fibration $\rho: V \rightarrow B$, where $B$ is a smooth complete curve. Let $\ell$ be a general fiber of $\rho$. We shall show that $\ell^{g}$ is a fiber of $\rho$ as well. In fact, note that $\left(C_{1}+C_{1}^{\prime} \cdot C_{i}+C_{i}^{\prime}\right)=0$ for every $1 \leq i \leq r$ and the $C_{i}+C_{i}^{\prime}(1 \leq i \leq r)$ exhaust all the $G$-translates of $C_{1}+C_{1}^{\prime}$. Hence $C_{i}+C_{i}^{\prime}$ is a fiber of $\rho$. Since

$$
\begin{aligned}
\left(\ell^{g} \cdot C_{1}+C_{1}^{\prime}\right) & =\left(\ell \cdot\left(C_{1}+C_{1}^{\prime}\right)^{g^{-1}}\right) \\
& =\left(\ell \cdot C_{i}+C_{i}^{\prime}\right)=0
\end{aligned}
$$

for some $i$, we know that $\ell^{g}$ is a fiber of $\rho$.

(2) If $\left(C_{1} \cdot C_{i}\right)=0$ for every $i \neq 1$, then $\left(C_{i}{ }^{2}\right)=0$ for $1 \leq i \leq s$ and a complete linear system $\left|N C_{1}\right|$ with $N \gg 0$ defines a $\mathbf{P}^{\mathbf{l}}$-fibration $\rho: V \rightarrow B$. It is clear that the $G$-action on $V$ preserves the $\mathbf{P}^{1}$-fibration $\rho$.

Q.E.D.

Theorem 1.7 With the above notations and assumptions, suppose that $(\bar{V}, \bar{\Delta})$ is $G$-relatively minimal. Let $\bar{F}$ be a G-extremal curve such that $\left(\bar{F}^{2}\right)=0$ and let $\bar{\rho}: \bar{V} \rightarrow B$ be the $\mathbf{P}^{1}$ fibration defined by a linear system $|n \bar{F}|$ for $n \gg 0$ (cf. Lemmas 1.3 and 1.6). Let $\bar{R}$ be $a$ fiber $\vec{\rho}$. Then the following assertions hold.

(1) A multiple of $\vec{R}$ is written in the form $\sum_{g \in G} \bar{L}^{g}$ up to the algebraic equivalence, where $\bar{L}$ is an irreducible curve.

(2) If $\bar{R}$ is reducible and there are no singular points lying on $\bar{R}$, then $\bar{R}$ consists of two $(-1)$ curves.

(3) If $\bar{R}$ is irreducible and reduced, then $\bar{R}$ is a smooth fiber. If $\bar{R}$ is non-reduced, there is a singular point lying on $\bar{R}$.

Proof. (1) Since $\bar{R}$ is numerically equivalent to a $Q$-multiple of $\bar{F}$, a multiple of $\bar{R}$ is a divisor of the form $\sum_{g \in G} \bar{L}$, where $\bar{L}$ is an irreducible curve.

(2) Write $\bar{R}=\sum_{i=1}^{s} \bar{L}_{i}$. If $s \geq 2$, then $\left(\bar{L}_{i}^{2}\right)<0$ for every $i$ because $\bar{R}$ is connected. Furthermore, the assertion (1) implies that $\left(\bar{L}_{i} \cdot \bar{\Delta}+K_{\bar{V}}\right)<0$ because $\left(\bar{F} \cdot \bar{\Delta}+K_{\bar{V}}\right)<0$. If 
there are no singular points lying on $\bar{R}$, the arguments in Lemma 1.6 shows that $\bar{R}$ consists of two $(-1)$ curves.

(3) If $\bar{R}$ is irreducible and if there are singular points lying on $\bar{R}$, the proper trnsform $R:=f^{\prime}(\bar{R})$ is a unique $(-1)$ curve in a fiber $f^{*}(\bar{R})$ of the $\mathbf{P}^{1}$-fibration $\bar{\rho} \cdot f: V \rightarrow C$. So, the multiplicity of $R$ is greater than 1. Te assertion (3) follows from this observation. Q.E.D.

We shall next consider the case $\rho(\bar{V} / / G)=1$. Then $\overline{\mathrm{NE}}(\bar{V})^{G}=\mathbf{R}_{+}[\bar{F}]$ with $\left(\bar{F}^{2}\right)>0$. Then $\bar{W}=\bar{V} / / G$ is a $\log$ del Pezzo surface of rank one. If $\bar{\Delta} \neq 0$, then $(\bar{W}, \bar{\Gamma})$ is an open $\log$ del Pezzo surface of rank one. Hence $\bar{W}-\bar{\Gamma}$ is isomorphic to $\mathrm{A}^{2} / \tilde{G}$, where $\widetilde{G}$ is a small finite subgroup of GL $(2, k)$. If $\bar{\Delta}=0$, then $\bar{V}$ is a complete log del Pezzo surface of rank one.

EXAMPLE 1.8 Let $\widetilde{G}$ be a small finite subgroup of $\mathrm{GL}(2, k)$, which is a central extension

$$
0 \longrightarrow C_{a} \longrightarrow \widetilde{G} \longrightarrow G \longrightarrow(1)
$$

where $C_{a}$ is a cyclic group of order $a$ and $G$ is a finite subgroup of SL $(2, k)$. The natural action of $\widetilde{G}$ on $\mathbf{A}^{2}$ via $\mathrm{GL}(2, k)$ extends to an action of $\widetilde{G}$ onto $\mathbf{P}^{2}$, where $\mathbf{A}^{2}$ is embedded into $\mathrm{P}^{2}$ by $(x, y) \mapsto(1, x, y)$. Let $H_{0}$ be the hyperplane at infinity. Then $H_{0}$ is $\widetilde{G}$-stable. Set $\bar{V}=\mathbf{P}^{2} / C_{a}$ and let $\bar{\Delta}$ be the image of $H_{0}$ on $\bar{V}$. Then $(\bar{V}, \bar{\Delta})$ is an open log del Pezzo surface of rank one with $G$-action. The quotient $\bar{W}=\bar{V} / / G$ is a completion of $\mathbf{A}^{2} / \widetilde{G}$.

EXAMPLE 1.9 Let $\bar{V}$ be the Hirzebruch surface $\mathbf{P}^{1} \times \mathbf{P}^{1}$. Let $\iota$ be the involution on $\bar{V}$ which exchanges the $\mathbf{P}^{1}$-factors, $(P, Q) \stackrel{\iota}{\longrightarrow}(Q, P)$, for $P, Q \in \mathbf{P}^{1}$. Then the quotient $\bar{W}=\bar{V} / /\langle\iota\rangle$ is isomorphic to $\mathbf{P}^{2}$, and the quotient morphism $\pi: \bar{V} \rightarrow \bar{W}$ branches over a conic $\bar{\Gamma}$ on $\mathbf{P}^{2}$. Let $\bar{\Delta}$ be the diagonal on $\bar{V}$ such that $\pi^{*}(\bar{\Gamma})=2 \bar{\Delta}$. Then $(\bar{W}, \bar{\Gamma})$ is a log del Pezzo surface of rank one, while rank Pic $(\bar{V})=2$.

\section{The case of relatively minimal $\mathrm{P}^{1}$-fibrations}

Consider the following example.

EXAMPLE 2.1 Let $W_{0}$ be the Hirzebruch surface of degree $n$ and let $M_{0}$ (resp. $M_{1}$ ) be the minimal cross-section (resp. a cross-section disjoint from $M_{0}$ ). Let $P_{0}$ be a point on $M_{1}$ and let $\ell_{0}$ be the fiber passing through $P_{0}$. Blow up the point $P_{0}$ and its infinitely near points $P_{1}, \ldots, P_{m-1}$ lying on the fiber $\ell_{0}$. Let $\sigma: W \rightarrow W_{0}$ be the composite of these blowing-ups. Let $E_{i}(1 \leq i \leq m)$ be the proper transform of the exceptional curve arising from the blowingup of the point $P_{i-1}$. Let $L_{0}$ be the proper transform of $\ell_{0}$. Then $L_{0}+E_{1}+2 E_{2}+\cdots+(m-$ 1) $E_{m-1}+m E_{m}$ is the total transform of the fiber $\ell_{0}$, whose dual graph is a linear chain with $\left(E_{i}^{2}\right)=-2$ for $1 \leq i \leq m-1,\left(E_{m}{ }^{2}\right)=-1$ and $\left(L_{0}{ }^{2}\right)=-m$. Then we can contract the curve $L_{0}$ and the curves $E_{1}+\cdots+E_{m-1}$ to the cyclic quotient singular points $Q_{0}$ and $Q_{1}$, respectively. Let $\tau: W \rightarrow \bar{V}$ be the contraction. Let $\bar{M}_{0}=\tau\left(M_{0}\right)$ and $\bar{M}_{1}=\tau\left(M_{1}\right)$. Then $\bar{V}$ has a $\mathbf{P}^{1}$-fibration $\bar{\rho}: \bar{V} \rightarrow \bar{C}$, where $\bar{C} \cong \mathbf{P}^{1}$. The curves $\bar{M}_{0}$ and $\bar{M}_{1}$ are the cross-sections of $\bar{\rho}$, and the point $Q_{i}$ lies on $\bar{M}_{i}$ for $i=0,1$.

Choose another fiber $\ell_{1}$ of the surface $W_{0}$. Then we have a linear equivalence

$$
L+E_{1}+2 E_{2}+\cdots+(m-1) E_{m-1}+(m-1) \sigma^{*}\left(\ell_{1}\right) \sim m\left(\sigma^{*}\left(\ell_{1}\right)-E_{m}\right) .
$$


So, we can consider a degree $m$ cyclic covering $\alpha: \widetilde{W} \rightarrow W$ which ramifies over $L+E_{1}+$ $\cdots+E_{m-1}+\sigma^{*}\left(\ell_{1}\right)$. Let $\mu: \widetilde{V} \rightarrow \widetilde{W}$ be the minimal resolution of the singularities. Let $\widetilde{L}_{0}$ and $\widetilde{E}_{m}$ be respectively the reduced inverse images of $L_{0}$ and $E_{m}$. Then $\left(\widetilde{L}_{0}^{2}\right)=-1$ and $\left(\widetilde{E}_{m}^{2}\right)=-m$. In fact, we can show that $(\alpha \cdot \mu)^{-1}\left(E_{1}+\cdots+E_{m-1}\right)$ and $\widetilde{L}_{0}$ are contractable to smooth points. Let $\nu: \widetilde{V} \rightarrow V$ be the contraction. Then $V$ has a $\mathbf{P}^{1}$-fibration $\rho: V \rightarrow C$ such that $R_{i}:=\alpha^{*}\left(\sigma^{\prime}\left(M_{i}\right)\right)$ is a cross-section of $\rho$ for $i=1,2$ and $C$ is an m-ple covering of $\bar{C}$ totally ramifying over the points $\bar{\rho}\left(\ell_{i}\right)(i=0,1)$. The cyclic group $G$ of order $m$ acts on $\widetilde{V}$ as the covering transformation group and the action of $G$ descends down to $V$. By the above construction, we know that the quotient surface $V / / G$ is isomorphic to $\bar{V}$.

We can consider the degenerate fibers of the same type $L_{0}^{(i)}+E_{1}^{(i)}+2 E_{2}^{(i)}+\cdots+(m-$ 1) $E_{m-1}^{i}+m E_{m}^{i}$ for $i=1, \ldots, r$ and the smooth fibers $\sigma^{*}\left(\ell_{j}\right)$ for $j=r+1, \ldots, r+s$, where $r+s$ is an even integer. Then the curve $C$ above is a smooth projective curve of genus $(m-1)(r+s-2) / 2$.

A $\mathbf{P}^{1}$-fibration $\rho: V \rightarrow C$ from a smooth projective surface $V$ with a finite group $G$-action is called $G$-relatively minimal if $G$ preserves the fibration $\rho$ and if every fiber is irreducible unless it consists of two $(-1)$ curves $C+C^{\prime}$ with $C^{\prime}=C^{g}$ for some $g \in G$. The next result shows that this example is essentially the unique case of a $G$-relatively minimal $\mathbf{P}^{1}$-fibration with a horizontal irreducible component of $D$ and a finite group $G$ acting only along fibers.

Theorem 2.2 Let $(V, D)$ be a pair of a smooth projective surface with a $\mathbf{P}^{1}$-fibration $\rho$ : $V \rightarrow C$ and a reduced effective divisor $D$ with simple normal crossings. Suppose that a finite group $G$ acts in such a way that $G$ preserves the $\mathbf{P}^{1}$-fibration $\rho$ and that $(V, \rho)$ is relatively minimal in the above sense. Suppose furthermore that $D$ contains horizontal components. Then the following assertions hold.

(1) The $\mathbf{P}^{1}$-fibration $\rho: V \rightarrow C$ is a $\mathbf{P}^{1}$-bundle. Namely, there are no fibers consisting of two $(-1)$ curves.

(2) There is only one horizontal component, say $R$, of $D$ which is not a component of $\mathrm{Bk} D, G$-stable and a cross-section of $\rho$.

(3) Let $\alpha: G \rightarrow$ Aut $C$ be the natural group homomorphism. If $\alpha$ is injective, the quotient surface $\bar{V}:=V / / G$ is a normal projective surface with $a \mathbf{P}^{1}$-bundle over $\bar{C}:=C / / G$ and $V$ is isomorphic to the normalization of the fiber product $\bar{V} \times{ }_{\bar{C}}(C, q)$, where $q: \bar{C} \rightarrow C$ is the quotient morphism.

(4) Let $H$ be the kernel of the homomorphism $\alpha$. Then $H$ is a cyclic group. Let $V_{1}:=V / / H$ be the quotient surface. Then $V_{1}$ is a smooth projective surface with a $\mathbf{P}^{1}$-fibration $\rho_{1}: V_{1} \rightarrow C$. The quotient morphism $p_{1}: V \rightarrow V_{1}$ is a cyclic covering which totally ramifies over the two cross-sections of the $\mathbf{P}^{1}$-fibration $\rho_{1}$.

Proof. (1) Suppose that $F=C+C^{\prime}$ is a fiber of $\rho$ consisting of two $(-1)$ curves. Let $R$ be a horizontal component of $D$. If $R \not \subset$ Supp Bk $D$, then $R^{g} \not \subset$ Supp Bk $D$ for any $g \in G$. Since $C^{\prime}=C^{g}$ for some $g \in G$, it follows that $\left(C^{\prime} \cdot R\right)>0$ if $R$ is $G$-stable and $\left(C^{\prime} \cdot R^{g}\right)>0$ 
for some $g \in G$ if $R$ is not $G$-stable. Since $D^{\#}+K_{V}$ is $G$-invariant, we have

$$
\begin{aligned}
|G|\left(F \cdot D^{\#}+K_{V}^{\prime}\right) & =\left(\sum_{g \in G} F^{g} \cdot D^{\#}+K_{V}\right) \\
& =\sum_{g \in G}\left\{\left(C \cdot D^{\#}+K_{V}\right)+\left(C^{\prime} \cdot D^{\#}+K_{V}\right)\right\} \\
& \geq \sum_{g \in G}\left\{\left(C \cdot R+K_{V}^{\prime}\right)+\left(C^{\prime} \cdot R^{g}+K_{V}\right)\right\} \\
& \geq 0 .
\end{aligned}
$$

This is a contradiction because $\sum_{g \in G} F^{g}$ gives rise to an extremal ray of $\overline{\mathrm{NE}}(\bar{V})^{G}$. Hence any horizontal component $R$ of $D$ is a component of $B k D$. Let $\left\{R_{i}\right\}_{i \in I}$ be the set of all irreducible components of $\mathrm{Bk} D$. Define the rational numbers $\alpha_{i}$ by the condition:

$$
\left(F+\sum_{i \in I} \alpha_{i} R_{i} \cdot R_{i}\right)=0 \quad \text { for every } i \in I .
$$

Then $\sum_{i \in I} \alpha_{i} R_{i}$ is a nonzero effective divisor because the intersection matrix of $\mathrm{Bk} D$ is negative definite and $\mathrm{Bk} D$ contains at least one horizontal component. Furthermore, the coefficient $\alpha_{i}>0$ if $\left(R_{i} \cdot F\right)>0$. Then we have

$$
\left(\bar{F}^{2}\right)=\left(\left(F+\sum_{i \in I} \alpha_{i} R_{i}\right)^{2}\right)=\left(F \cdot F+\sum_{i \in I} \alpha_{i} R_{i}\right)>0,
$$

which contradicts the hypothesis that $\left(\bar{F}^{2}\right)=\left(F^{2}\right)=0$. Hence $\rho$ is a $\mathbf{P}^{1}$-bundle.

(2) By (1) above, any fiber $F$ of $\rho$ is irreducible. If there is an irreducible horizontal component of $\mathrm{Bk} D$, then $\left(\bar{F}^{2}\right)>0$, which is a contradiction. Suppose that there exists an irreducible component $R$ of $D$ such that $(R \cdot F) \geq 2$. Then we have

$$
\begin{aligned}
|G|\left(F \cdot D^{\#}+K_{V}\right) & =\left(\sum_{g \in G} F^{g} \cdot D^{\#}+K_{V}\right) \\
& \geq \sum_{g \in G}\left(F^{g} \cdot R^{g}+K_{V}\right) \\
& \geq 0,
\end{aligned}
$$

which implies the stated results.

(3) Let $Y=V-R$, where $R$ is the unique $G$-stable cross-section of $\rho$. By (2) above, $Y$ is $G$-stable and $\left.\rho\right|_{Y}: Y \rightarrow C$ is an $\mathbf{A}^{1}$-bundle. We denote $\left.\rho\right|_{Y}$ by the same letter $\rho$. Since $G$ preserves the $\mathbf{A}^{1}$-fibration $\rho: Y \rightarrow C$, there exists a natural group homomorphism $\alpha: G \rightarrow$ Aut $C$ such that $\rho(v)^{\alpha(g)}=\rho\left(v^{g}\right)$ for $g \in G$ and $v \in V$. Let $K^{\prime}$ be the function field of $C$ over the ground field $k$ and let $\bar{K}$ be the function field of $\bar{C}$ over $k$, where $\bar{C}=C / / G$. Since the generic fiber of $\rho$ is the affine line $\mathbf{A}_{K}^{1}:=\operatorname{Spec} K[x]$, we have

$$
g(x)=a(g) x+b(g) \text { with } a(g) \in K^{*}, b(g) \in K^{\prime} \text { for } g \in G,
$$

where

$$
a(g h)=a(h)^{g} a(g) \quad \text { and } \quad b(g h)=a(h)^{g} b(g)+b(h)^{g} \quad \text { for } \quad g, h \in G .
$$


Suppose that $\alpha$ is injective. Then $G$ is considered as the Galois group of a field extension $K / \bar{K}$. By Theorem 90 of Hilbert, there exists $c \in K^{*}$ such that $a(g)=c \cdot\left(c^{g}\right)^{-1}$. Replacing $x$ by $c x$, we nay assume that $a(g)=1$. Then $b(g)=\left(d-d^{g}\right) /|G|$, where $d=\sum_{g \in G} b(g)$. Replacing $x$ by $x+d$, we may assume that $b(g)=0$ as well. Hence, with $x$ chosen this way, we have $g(x)=x$ for $g \in G$.

It then follows that there exists a $G$-stable $U$ open set of $C$ such that $\rho^{-1}(U) \cong U \times \mathbf{P}^{1}$ with $G$ acting on the factor $U$. Let $\bar{V}:=V / / G$, which is a normal projective surface with the morphism $\bar{\rho}: \bar{V} \rightarrow \bar{C}$, where $\bar{C}:=C / / G$. Let $p: V \rightarrow \bar{V}$ and $q: C \rightarrow \bar{C}$ be the quotient, morphisms. Then $\bar{\rho} \cdot p=q \cdot \rho$. The above observation implies that $\bar{\rho}^{-1}(\bar{U}) \cong \bar{U} \times \mathbf{P}^{1}$, where $\bar{U}=U / / G$. Hence $\bar{\rho}: \bar{V} \rightarrow \bar{C}$ is a $\mathbf{P}^{\mathbf{l}}$-fibration. Let $\bar{R}:=R / / G$. Since $R$ is a cross-section of $\rho$, it follows that $\bar{R}$ is a cross-section of $\bar{\rho}$, i.e., $\left.\bar{\rho}\right|_{\bar{R}}$ is the identity morphism. By [3], the singularities of $\bar{V}$ which are not on the cross-section $\bar{R}$ are cyclic quotient singularities, for $\bar{V}-\bar{R}$ contains an $\mathbf{A}^{1}$-cylinder. It is clear that the natural morphism $V \rightarrow \bar{V} \times_{\bar{C}} C$ is a finite birational morphism. Hence $V$ is isomorphic to the normalization of the fiber product $\bar{V} \times \bar{C} C$.

Note that if a fiber $\bar{F}$ has a singular point $Q$ which is not on the cross-section $\bar{R}$, then $\bar{F}$ has also a singular point on the point $\bar{F} \cap \bar{R}$. In fact, let $Q$ be a singular point of $\bar{V}$ not lying on $\bar{R}$ if it exists at all. Suppose that $\bar{F} \cap \bar{R}$ is a smooth point of $\bar{V}$. Since $\rho: V \rightarrow C$ is a $\mathbf{P}^{1}$-bundle, $\bar{F}$ is irreducible. Let $\sigma: W \rightarrow \bar{V}$ be the minimal resolution of singularities. Then $\sigma^{-1}(Q)$ consists of rational curves, each component of which has self-intersection number $\leq-2$. Hence $\sigma^{-1}(\bar{F})$ consists of the proper transform $F^{\prime}$ of $\bar{F}$ and the rational curves with self-intersection number $\leq-2$. Since $\sigma^{-1}(\bar{F})$ is a degenerate fiber of a $\mathbf{P}^{1}$-fibration $\bar{\rho} \cdot \sigma: W \rightarrow \bar{C}$, the component $F^{\prime}$ is the unique $(-1)$ component. Since $\bar{F}$ meets the crosssection $\bar{R}$ and since $\bar{F} \cap \bar{R}$ is a smooth point of $\bar{V}, F^{\prime}$ has multiplicity 1 in the fiber $\sigma^{-1}(\bar{F})$. Then there exists another $(-1)$ component in $\sigma^{-1}(\bar{F})$. This is a contradiction.

(4) The correspondence $g \mapsto a(g)$ induces a group homomorphism $a: H \rightarrow G_{m}$, where $H=\operatorname{Ker} \alpha$. If the homomorphism is not injective, then we have, with the above notations, $g(x)=x+b(g)$ for $g \in \operatorname{Ker} a$. Then $g \in \operatorname{Ker} a$ is of infinite order provided $g \in \operatorname{Ker} a$ and $g \neq 1$. This is a contradiction. Hence Ker $a=(1)$. So, $a$ is injective. Then, as a finite subgroup of $G_{m}, H$ is a cyclic group of order, say $n$. Let $g$ be a generator of the group $H$. Then $a(g)$ is an $n$-th primitive root of unity. Suppose $H \neq(1)$. Then $g(x)=a(g) x+b(g)$ and $g(x+c)=a(g)(x+c)$, where $c=b(g) /(a(g)-1)$. Then the point $x=-c$ is left fixed under the $H$-action. The stated assertion then follows immediately.

Q.E.D.

We consider next the case where the boundary divisor $D$ has no horizontal components with respect to the $\mathbf{P}^{1}$-fibration $\rho: V \rightarrow C$. Our objective is to prove Theorem 2.6 .

Lemma 2.3 Let $\rho: V \rightarrow C$ be a G-relatively minimal $\mathbf{P}^{1}$-fibration. Suppose that $D$ has no horizontal components and that $\rho$ contains a fiber of the type $F=C+C^{\prime}$, where $C^{\prime}=C^{g}$ for $g \in G$. Then the following assertions hold:

(1) $C=C^{\prime g}$ and $C \cap C^{\prime}$ is fixed under the action of $g$.

(2) If $g$ acts along the fibers, i.e., $\rho \cdot g=\rho$, then $g$ has order 2 or 4 .

(3) If $g^{2}$ moves the fibers, i.e., $\rho \cdot g \neq \rho$, then $g^{2}$ has order either $n=2(s+1)$ for some $s>0$ or $n=3$. 
Proof. (1) Suppose that $C^{g}$ is contained in a fiber different from $F$. Since $C^{g}$ is contained in the same fiber as $C^{\prime g}$, it follows that $C^{\prime g}$ is contained in the fiber $F$. Hence $C^{\prime g}=C$. Let $P:=C \cap C^{\prime}$. Then $P^{g} \in C^{g} \cap C^{\prime g}=C^{\prime} \cap C=\{P\}$, whence we know that $P$ is fixed under the action of $g$.

(2) The proof of the assertion consists of three steps.

(I) By the assertion (1), we have $C^{g^{2}}=C$ and $C^{\prime g^{2}}=C^{\prime}$. Set $h=g^{2}$. Suppose that $h$ is not the identity element. Then $h$ is an automorphism of $\mathbf{A}^{1}=C-\{P\}$. Let $x$ be an inhomogeneous coordinate of $\mathbf{A}^{1}$, with respect to which the point $P$ corresponds to $x=\infty$. Then $h(x)=\alpha x+\beta$ with $\alpha \in k^{*}$ and $\beta \in k$. Let $n$ be the order of $h$. It is then easy to show that

$$
\alpha^{n}=1 \text { and }\left(\alpha^{n-1}+\cdots+\alpha+1\right) \beta=0 .
$$

Let $\gamma=\beta /(\alpha-1)$. Then $h(x+\gamma)=\alpha(x+\gamma)$. So, by replacing $x$ by $x+\gamma$, we may assume that $h(x)=\alpha x$. This implies that the components $C$ and $C^{\prime}$ have the points $Q$ and $Q^{\prime}$ respectively which are fixed under $h$.

(II) In an open neighborhood of the point $P$, we can choose a system of local coordinates $(x, y)$ such that $C$ (resp. $C^{\prime}$ ) is defined by $y=0$ (resp. $x=0$ ) and that $h(x)=\alpha x$ and $h(y)=\alpha y$. Here $h$ acts on $x$ and $y$ with the same weight because $C$ and $C^{\prime}$ are interchanged by the action of $g$. Let $q: V \rightarrow \widehat{V}$ be the quotient morphism under the group $\langle h\rangle$. Then $\widehat{V}$ has a $\mathbf{P}^{1}$-fibration $\widehat{\rho}: \widehat{V} \rightarrow \widehat{C}$ because $h$ preserves the $\mathbf{P}^{1}$-fibration $\rho$. Set $\widehat{P}=q(P), \widehat{Q}=q(Q)$ and $\widehat{Q}^{\prime}=q\left(Q^{\prime}\right)$. Then the branch locus of $q$ contains these three points $\widehat{P}, \widehat{Q}$ and $\widehat{Q}^{\prime}$. Meanwhile, $\widehat{V}$ has an isolated cyclic quotient singularity at $\widehat{P}$. Hence $\widehat{P}$ is the isolated component of the branch locus of $q$. The minimal resolution of $\widehat{P}$ consists of only one $(-n)$ curve $A$ and the proper transforms of $q(C), q\left(C^{\prime}\right)$ (say, $\widehat{C}, \widehat{C}^{\prime}$ ) meet the component $A$ transversally.

(III) On the other hand, the points $\widehat{Q}$ and $\widehat{Q}^{\prime}$ are smooth points, for the cyclic group $\langle h\rangle$ acts on $V$ near $Q$ as $h(\xi, \eta)=\left(\alpha^{-1} \xi, \eta\right)$ with respect to a suitable local system of parameters $(\xi, \eta)$. Hence $\widehat{C}+A+\widehat{C}^{\prime}$ is a degenerate fiber of the $\mathbf{P}^{1}$-fibration on a smooth surface. This implies that $\widehat{C}$ and $\widehat{C}^{\prime}$ are $(-1)$ curves. Hence $n=2$. Hence the order of $g$ is 4 .

(3) Suppose that $h$ moves the fibers. Then the points $\widehat{Q}$ and $\widehat{Q}^{\prime}$ are the isolated fixed points of $h$. Suppose that $h$ acts on $V$ near $Q$ as $h(\xi, \eta)=\left(\alpha^{-1} \xi, \alpha^{-d} \eta\right)$ with $0<d<n$, where $(\xi, \eta)$ is a suitable local system of parameters at $Q$. Let $e=\operatorname{gcd}(n, d)$ and let

$$
\frac{n}{d}=\beta_{1}-\frac{1}{\beta_{2}-\frac{1}{\ddots \quad-\frac{1}{\beta_{s}}}}
$$

be the continued fraction expansion of $(n / \epsilon) /(d / e)$. Then the minimal resolution of three singular points $\widehat{P}, \widehat{Q}, \widehat{Q}^{\prime}$ together with $\widehat{C}, \widehat{C}^{\prime}$ give rise to a degenerate $\mathbf{P}^{1}$-fiber whose dual graph is given as follows. 


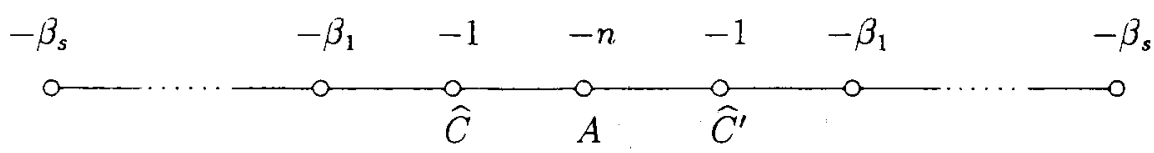

Hence either $\beta_{1}=\cdots=\beta_{s}=2$ and $n=2(s+1)$ or $n=\beta_{1}=3$ and $s=1$. In the first case, $n / e=s+1$ and $d / e=s$. Hence $e=2, n=2(s+1)$ and $d=2 s$. In the second case, $n=3$ and $d=1$.

Q.E.D.

Lemma 2.4 In Lemma 2.3, the case where $g^{2}$ has order 3 is impossible.

Proof. The element $g$ induces an involution $\widehat{g}$ on $\widehat{V}$ which preserves the $\mathbf{P}^{1}$-fibration $\hat{\rho}$ and interchanges $\widehat{C}$ and $\widehat{C}^{\prime}$. Hence $\widehat{g}$ fixes the point $\widehat{P}$ and interchanges the points $\widehat{Q}$ and $\widehat{Q}$. Hence $\widehat{g}$ lifts to an involution $\widetilde{g}$ on the minimal resolution $\sigma: \widetilde{V} \rightarrow \widehat{V}$. The $\mathbf{P}^{1}$-fibration $\hat{\rho}$ lifts to a $\mathbf{P}^{1}$-fibration $\tilde{\rho}: \widetilde{V} \rightarrow \widehat{C}$. The inverse image of the fiber $q\left(C+C^{\prime}\right)$ has the following linear chain:

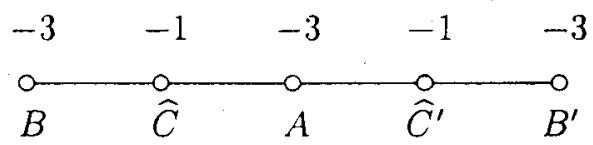

where we denote the proper transforms of $\widehat{C}$ and $\widehat{C}^{\prime}$ on $\widetilde{V}$ by the same letters. The exceptional curve $A$ arising from $\widehat{P}$ is stable under the action of $\widetilde{g}$. Since the points $\widehat{C} \cap A$ and $\widehat{C}^{\prime} \cap A$ interchages by $\widetilde{g}, A$ has two points $R, R^{\prime}$ which are fixed by $\widetilde{g}$. We consider two cases according as $\widetilde{g}$ moves the fibers of $\widetilde{\rho}$ or not. Let $\bar{V}$ be the quotient of $\widetilde{V}$ by $\widetilde{g}$ which has the $\mathbf{P}^{1}$-fibration $\bar{\rho}: \bar{V} \rightarrow \widetilde{C}$. Let $\bar{F}$ be the image of $\sigma^{-1}\left(q\left(C+C^{\prime}\right)\right)$. Suppose $\widetilde{g}$ moves the fibers of $\tilde{\rho}$. Then the points $R, R^{\prime}$ are mapped to the singular points $\bar{R}, \bar{R}^{\prime}$ of type $A_{2}$ on the surface $\bar{V}$. Then the minimal resolution of the points $\bar{R}, \bar{R}^{\prime}$ gives rise to a degenerate $\mathbf{P}^{1}$-fiber of the following type on a smooth surface:

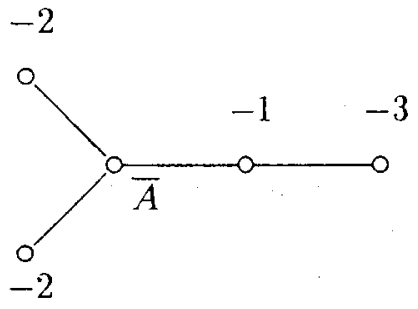

But, with whatever value for the self-intersection number $\left(\bar{A}^{2}\right)$, this graph cannot be the graph of a degenerate $\mathbf{P}^{1}$-fiber. Suppose that $\tilde{g}$ does not move the fibers of $\tilde{\rho}$. Then there are no singular points appearing on the fiber $\bar{F}$. Since $\left(A^{2}\right)=-3$ and $\left(A^{2}\right)=2\left(\bar{A}^{2}\right)$, this is impossible again.

Q.E.D.

Lemma 2.5 In Lemma 2.3, the following two cases do not occur.

(1) The element $g^{2}$ has order $n=2(s+1)$ with $s>0$. 
(2) The element $g$ has order 4.

Proof. (1) With the notations in Lemma 2.3, the points $Q, Q^{\prime}$ on $C, C^{\prime}$ are isolated fixed points under the action of $h:=g^{2}$. Hence the points $\widehat{Q}, \widehat{Q}^{\prime}$ on $\widehat{C}, \hat{C}^{\prime}$ are the isolated cyclic singular points on the surface $\widehat{V}$. The element $g$ induces an automorphism on $\widehat{V}$ which we denote by the same letter $g$. Then $g$ lifts to an automorphism $g$ on the minimal resolution $\widetilde{V}$ and interchanges the componerts $\widehat{C}, \widehat{C}^{\prime}$ as well as the linear chains sprouting from $\widehat{C}, \widehat{C}^{\prime}$.

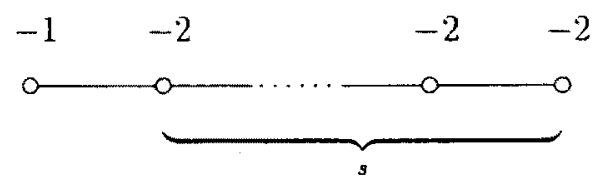

Furthermore, $g$ acts on the component $A$. Since $g$ interchanges the points $\widehat{C} \cap A, \hat{C}^{\prime} \cap A$ on $A$, there are two other points $R, R^{\prime}$ of $A$ which are left fixed by $g$. There are two cases according as $g$ moves the fibers of the $\mathbf{P}^{1}$-fibration $\tilde{\rho}: \widetilde{V} \rightarrow \widehat{C}$ or not.

CASE 1 . Consider first the case where $g$ moves the fibration $\tilde{\rho}$. Let $\bar{V}$ be the quotient of $\widetilde{V}$ by $g$. Then the images $\bar{R}, \bar{R}^{\prime}$ of $R, R^{\prime}$ are the cyclic singular points of type $A_{2}$. Let $W$ be the minimal resolution of $\bar{V}$. Then $W$ has a $\mathbf{P}^{1}$-fibration $\rho_{W}: W \rightarrow \bar{C}$, where $\bar{C}$ is the quotient of $\widehat{C}$ by an involution. The fiber of $\rho_{W}$ corresponding to $q\left(C+C^{\prime}\right)$ on $\widehat{V}$ has the following dual graph:

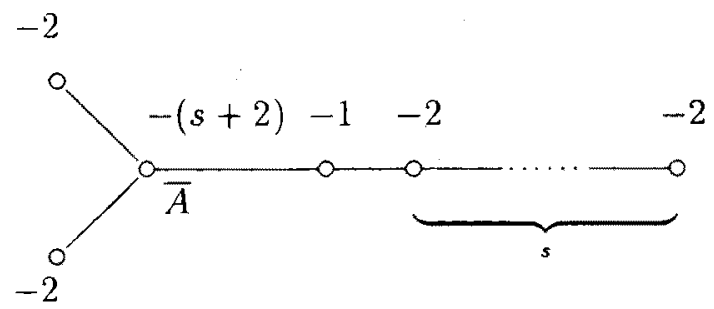

In fact, the surface $\widetilde{V}$ is obtained by taking a double covering of $W$ ramifying on the two $(-2)$ components meeting $\bar{A}$ and one more fiber of $\rho_{W}$ and by contracting the inverse images of the $(-2)$ components which become $(-1)$ components on the double covering.

Since the $\mathbf{P}^{1}$-fibation $\rho_{W}$ is trivial over an open set of $\bar{C}$, the function field $k(\widetilde{V})$ is written as $k(W)[t] /\left(t^{2}=x\right)$, where $x$ is an inhomogeneous parameter of $\bar{V}$ such that the above double covering ramifies over the fibers (the fiber components) of $\rho_{W}$ lying over $x=0, \infty$.

To go further, we have to look into the quotient morphism $q: V \rightarrow \widehat{V}$ more closely. With the notations of Lemma 2.3 , the element $h$ acts on $V$ near the point $Q$ as $h(\xi, \eta)=$ $\left(\alpha^{-1} \xi, \alpha^{-d} \eta\right)$, where $\alpha$ is a primitive $n$-th root of unity and $d=2 s$. Set $h^{\prime}=h^{s+1}$. Then $h^{\prime 2}=1$ and the element $h^{\prime}$ acts as $h^{\prime}(\xi, \eta)=(-\xi, \eta)$ near the point $Q$ (and hence near the point $\left.Q^{\prime}\right)$. Hence $h^{\prime}$ acts along the fibers of $\rho$, i.e., $\rho \cdot h^{\prime}=\rho$. Let $q_{1}: V \rightarrow \widehat{V}_{1}$ be the quotient morphism by $h^{\prime}$ and let $\widetilde{V}_{1}$ be the minimal resolution. The $\mathbf{P}^{l}$-fibration $\rho: V \rightarrow C$ descends to a $\mathbf{P}^{1}$-fibration $\widetilde{\rho}_{1}: \widetilde{V}_{1} \rightarrow C$ and the fiber $\widetilde{\rho}_{1}^{-1}\left(\rho\left(C+C^{\prime}\right)\right)$ has the following dual graph: 


\begin{tabular}{ccc}
-1 & -2 & -1 \\
\hdashline & - & - \\
$\widehat{C}_{1}$ & $\widehat{A}_{1}$ & $\hat{C}_{1}^{\prime}$
\end{tabular}

Let $\widehat{h}$ be the automorphism on $\widehat{V}_{1}$ and $\widetilde{V}_{1}$ induced by $h$. Then $\widehat{h}^{s+1}=1$ and $\widehat{h}$ acts trivially on the component $\widehat{A}_{1}$. Let $Q_{1}, Q_{1}^{\prime}$ be the images on $\widetilde{V}_{1}$ of the points $Q, Q^{\prime}$, respectively. Then $\hat{h}$ acts on $\widetilde{V}_{1}$ near the point $Q_{1}\left(\right.$ and $\left.Q_{1}^{\prime}\right)$ as $\hat{h}\left(\xi_{1}, \eta_{1}\right)=\left(\alpha_{1}^{-1} \xi_{1}, \alpha_{1}^{-s} \eta_{1}\right)$, where $\alpha_{1}$ is a primitive $(s+1)$-th root of unity and where $\left(\xi_{1}, \eta_{1}\right)$ is a system of local parameters such that $\widehat{C}_{1}$ is defined by $\eta_{1}=0$. The quotient of $\widehat{V}_{1}$ by the action of $\widehat{h}$ is the surface $\widehat{V}$. Hence the function field $k\left(\widehat{V}_{1}\right)$ is given as $k(\widehat{V}) \otimes_{k(\widehat{C})} k(C)$ with a cyclic Galois extension $k(C) / k(\widehat{C})$ of degree $s+1$ and the function field $k(V)$ is obtained as $k\left(\widehat{V}_{1}\right)[u] /\left(u^{2}=y\right)$, where $y$ is a fiber coordinate of the $\mathbf{P}^{1}$-fibration $\widetilde{\rho}_{1}$ over an open set of $C$. Thus the field extension $k(V) / k(\bar{V})$ is a composite of a cyclic Galois extension $k\left(\widehat{V}_{1}\right)=k(\bar{V}) \otimes_{k(\bar{C})} k(C)$ of degree $2(s+1)$ and a quadratic extension $k\left(\widehat{V}_{1}\right)[u] /\left(u^{2}=y\right)$. Since $k(V)=k(C)(u)$, the field extension $k(V) / k(\bar{V})$ cannot be a cyclic extension of degree $4(s+1)$.

CASE 2. Consider next the case where $g$ does not move the fibration $\widetilde{\rho}$. With the notations of Case 1 above, the $\mathbf{P}^{1}$-fibration $\widetilde{\rho}: \widetilde{V} \rightarrow \widehat{C}$ has two horizontal cross-sections which meet the fiber components $A$ in the points $R, R^{\prime}$. Hence the function field $k(\widehat{V})$ is a quadratic extension $k(\bar{V})[u] /\left(u^{2}=z\right)$, where $z$ is a fiber coordinate of the $\mathbf{P}^{1}$-fibration $\rho_{W}: \bar{V} \rightarrow \bar{C}$, where $\bar{C}=\widehat{C}$. Let $q_{2}: \widehat{V}_{1} \rightarrow \widehat{V}$ be the quotient morphism by the automorphism $\hat{h}$ induced by $h$. The same arguments as in the case 1 shows that the function field $k\left(\hat{V}_{1}\right)$ is written as $k(\widehat{V}) \otimes_{k(\widehat{C})} k(C)$, where $k(C) / k(\widehat{C})$ is a cyclic extension of degree $s+1$. Furthermore, $q_{2}$ induces an isomorphism between the comonents $\hat{A}_{1}$ and $A$. Let $R_{1}, R_{1}^{\prime}$ be the points of $\widehat{A}_{1}$ which correspond to the points $R, R^{\prime}$. We may assume that $u=0, \infty$ at the points $R, R^{\prime}$, respectively. Hence, if we consider $u$ as the inhomogeneous coordinate of $\hat{A}_{1}$ via $q_{2}, u$ takes the value $0, \infty$ at the points $R_{1}, R_{1}^{\prime}$, respectively. Note that the points $R_{1}, R_{1}^{\prime}$ are different from the points $\widehat{C}_{1} \cap \widehat{A}_{1}, \widehat{C}_{1}^{\prime} \cap \widehat{A}_{1}$. So, we may assume that $u=1$ (resp. -1 ) at the point $\widehat{C}_{1} \cap \widehat{A}_{1}$ (resp. $\widehat{C}_{1}^{\prime} \cap \widehat{A}_{1}$ ). The double covering $q_{1}: V \rightarrow \widehat{V}_{1}$ ramifies over $\widehat{A}_{1}$ and the two cross-sections of $\widetilde{\rho}_{1}$ which meet the fiber $\widehat{C}_{1}+\widehat{A}_{1}+\widehat{C}_{1}^{\prime}$ at the points $Q_{1}, Q_{1}^{\prime}$ on the components $\widehat{C}_{1}, \widehat{C}_{1}^{\prime}$, respectively. Hence the field extension $k(V) / k\left(\widehat{V}_{1}\right)$ is given as

$$
k(V)=k\left(\widehat{V}_{1}\right)[v], \quad \text { where } v^{2}=\frac{u+1}{u-1} .
$$

Hence, replacing $v$ by $(u-1) v$, we may assume that $v^{2}=u^{2}-1=z-1$. So, we have

$$
k(V)=\left(k(\bar{V}) \otimes_{k(\widehat{C})} k(C)\right)[u, v] /\left(u^{2}=z, v^{2}=z-1\right) .
$$

This implies that the Galois group of the extension $k(V) / k(\bar{V})$ is isomorphic to $\mathbf{Z}_{1} \times \mathbf{Z}_{2} \times \mathbf{Z}_{s+1}$, which is not a cyclic group of order $4(s+1)$.

(2) By the proof of Lemma 2.3, this is the case 2 above, where the quotient morphism $q_{2}: \widehat{V}_{1} \rightarrow \widehat{V}$ is the identity morphism. Hence $k(V)=k(\bar{V})[u, v] /\left(u^{2}=z, v^{2}=z-1\right)$, and 
the Galois group of the extension $k(V) / k(\bar{V})$ is isomorphic to $\mathbf{Z}_{2} \times \mathbf{Z}_{2}$. So, it is not a cyclic group of order 4.

Q.E.D.

As a consequence of Lemmas 2.3, 2.4 and 2.5, we obtain the following result.

Theorem 2.6 Let $\rho: V \rightarrow C$ be a relatively minimal $\mathbf{P}^{1}$-fibration with a $G$-action. Suppose that $D$ has no horizontal components and that $\rho$ contains a fiber of the type $F=C+C^{\prime}$, where $C^{\prime}=C^{g}$. Then $g$ is an involution.

EXAMPLe 2.7 Let $V_{0}$ be the Hirzebruch surface of degree $n \geq 2$ and let $M$ be the minimal section. Let $B$ be a smooth irreducible curve which is linearly equivalent to $2 M+2 n \ell$, where $\ell$ is a fiber of the canonical $\mathbf{P}^{1}$-fibration $\rho_{0}$ on $V_{0}$. Then $B$ has genus $n-1$. Hence the restriction $\left.\rho_{0}\right|_{B}: B \rightarrow \mathbf{P}^{1}$ ramifies at $2 n$ points on $B$. Let $\sigma: V \rightarrow V_{0}$ be the double covering ramified over the curve $B$ and let $\iota: V \rightarrow V$ be the covering involution.

Let $\ell_{1}, \ldots, \ell_{2 n}$ be the fibers of $\rho_{0}$ which meet the curve $B$ only in single points with multiplicity 2. Then $\sigma^{*}\left(\ell_{i}\right)=C_{i}+C_{i}^{\prime}(1 \leq i \leq 2 n)$, where $C_{i}$ and $C_{i}^{\prime}$ are the $(-1)$ curves meeting each other transversally in one point. The composite $\rho:=\rho_{0} \cdot \sigma: V \rightarrow \mathbf{P}^{1}$ is a $\mathbf{P}^{1}$-fibration and has $C_{i}+C_{i}^{\prime}$ as a degenerate fiber. The involution $\iota$ exchanges $C_{i}$ and $C_{i}^{\prime}$.

Since $B \cap M=\emptyset$, the inverse image $\sigma^{*}(M)$ is a disjoint sum $M_{0}+M_{1}$ with $\left(M_{0}{ }^{2}\right)=$ $\left(M_{1}{ }^{2}\right)=-n$. We may assume that the $C_{i}$ meet $M_{0}$ and the $C_{i}^{\prime}$ meet $M_{1}$. The contraction of $C_{1}^{\prime}, \ldots, C_{2 n}^{\prime}$ brings the surface $V$ back again to the Hirzebruch surface $V_{0}$, The image of $M_{1}$ is the section $M^{\prime}$ of $V_{0}$ which is disjoint from the minimal section $M$. Let $\tau: V \rightarrow V_{0}$ be the contraction of $C_{1}^{\prime}, \ldots, C_{2 n}^{\prime}$. Then we have

$$
\begin{aligned}
K^{\prime} V_{0} & \sim-2 M_{0}-(n+2) \ell \\
K_{V} & \sim \tau^{*}\left(K_{V_{0}}\right)+\sum_{i=1}^{2 n} C_{i}^{\prime} \\
\tau^{*}\left(M^{\prime}\right) & =M_{1}+\sum_{i=1}^{2 n} C_{i}^{\prime} .
\end{aligned}
$$

Hence $K_{V} \sim-M_{0}-M_{1}-2 \ell$. Let $D=M_{0}+M_{1}$. Then we compute

$$
D^{\#}+K_{V}=\frac{n-2}{n} M_{0}+\frac{n-2}{n} M_{1}+K_{V}
$$

Then $C_{i}+C_{i}^{\prime}$ is a ı-invariant extremal curve with $\left(C_{i}+C_{i}^{\prime} \cdot D^{\#}+K_{V}^{\prime}\right)=-4 / n$.

It follows by induction that

$$
b\left(g^{m}\right)=\left(a(g)^{m-1}+\cdots+a(g)+1\right) b(g) \text { for } g \in \operatorname{Ker} \alpha
$$

where $b(1)=0$. If $a(g) \neq 1$, then the point given by $x=-b(g) /(a(g)-1)$ is left fixed by the action of $g$, but this is not possible because $G$ acts on $Y_{K}$ freely. If $a(g)=1$ and $m$ is the order of $g$, then $b(g)=0$ because $a(g)^{m-1}+\cdots+a(g)+1=m \neq 0$ and $b\left(g^{m}\right)=0$. Then $g$ acts on $\widetilde{Y}$ trivially, and this is a contradiction. Hence Ker $\alpha=(1)$ and $\alpha$ is injective. 


\section{Appendix by $M$. Namba}

The following result might be well-known among the experts.

Theorem 3.1 Let $X$ be a complete smooth curve of genus $g$ defined over the ground field $k$ of characteristic zero. Let $\varphi$ be a nontrivial automorphism of finite order. Let $d$ be the order of $\varphi$. Then the number of the fixed points of $\varphi$ is less than or equal to $2 g /(d-1)+2$. The equality holds if and only if $X$ and $\varphi$ are given as follows:

$$
X: \quad y^{d}=\left(x-a_{1}\right) \cdots\left(x-a_{s}\right) \quad \text { with } \quad s=\frac{2 g}{d-1}+2,
$$

where $a_{1}, \ldots, a_{s}$ are all distinct, and where $\varphi$ is given by

$$
(x, y) \mapsto(x, \zeta y)
$$

with a d-thprimitive root $\zeta$ of the unity.

Proof. Let $\langle\varphi\rangle$ be the cyclic group of order $d$ generated by the said automorphism $\varphi$. Let $Y$ be the quotient of $X$ by $\langle\varphi\rangle$ and $\pi: X \rightarrow Y$ be the quotient morphism. Let $g_{0}$ be the genus of the smooth curve $Y$. Let $B_{\pi}=\left\{q_{1}, \ldots, q_{s}\right\}$ be the branch locus of $\pi$. Then $\langle\varphi\rangle$ acts transitively on the set $\pi^{-1}\left(q_{i}\right)$ for $1 \leq i \leq s$. Let $e_{1}, \ldots, e_{s}$ be the respective ramification indices over the points $q_{1}, \ldots, q_{s}$. By the Riemann-Hurwitz formula, we then have

$$
2 g-2=d\left(2 g_{0}-2\right)+\sum_{i=1}^{s} \frac{d}{e_{j}}\left(e_{i}-1\right) .
$$

It is written as

$$
\sum_{i=1}^{s}\left(1-\frac{1}{e_{i}}\right)=\frac{2 g-2}{d}+2-2 g_{0} .
$$

Let $P$ be a point of $X$. Then $P$ is fixed by $\varphi$ if and only if $\varphi$ is totally ramified over the point $q:=\varphi(P)$, i.e., $q \in B_{\pi}$ and $d=e$ at $q$. Let $n$ be the number of the fixed points of $\varphi$. Then the equality (1) implies that

$$
n\left(1-\frac{1}{d}\right) \leq \frac{2 g-2}{d}+2 .
$$

So, we obtain the inequality

$$
n \leq \frac{2 g}{d-1}+2
$$

Now the above computations show that the equality occurs in the inequality (2) if and only if $\pi$ ramifies totally over every point of $B_{\pi}$ and the curve $Y$ has genus $g_{0}=0$. Hence the curve $X$ determines as follows:

$$
y^{d}=\left(x-a_{1}\right) \cdots\left(x-a_{n}\right), \quad \text { where } \quad n=\frac{2 g}{d-1}+2 .
$$

Here $(d-1) \mid 2 g$ because $s$ is an integer.

Q.E.D.

Now fix the genus $g$ and consider when the maximum value of $n$ is attained. 
Corollary 3.2 Let $X$ be a complete smooth curve of genus $g$ and let $\varphi$ be a nontrivial automorphism of $X$ of finite order. Then the number of the points left fixed by $\varphi$ is less than or equal to $2 g+2$. The number is equal to $2 g+2$ if and only if one of the following cases occur:

(1) $X$ is a hyperelliptic curve and $\varphi$ is the hyperelliptic involution.

(2) $X$ is an elliptic vurve and $\varphi$ is the multiplication by $(-1)$.

(3) $X$ is a rational curve and $\varphi(x)=\zeta x$, where $\zeta$ is a primitive $d$-th root of the unity and $x$ is an inhomogeneous coordinate.

Proof. The relation in (3) implies that the maximal value $2 g+2$ of $n$ is attained if and only if either $d=2$ and $g>0$ or $g=0$. If $d=2$ and $g \geq 2$, then $X$ is a hyperelliptic curve and $\varphi$ is the hyperelliptic involution. Suppose $g=1$ and $d=2$. Then the hyperelliptic involution is the multiplication by $(-1)$ when $X$ is defined by the equation in (3). If $g=0$ then $\varphi$ fixes two points $P_{1}, P_{2}$. Choose an inhomogeneous coordinate $x$ so that $x=0, \infty$ at $P_{1}, P_{2}$, respectively. Then $\varphi(x)=\zeta x$ for a primitive $d$-th root of unity.

Q.E.D.

We note that any automorphism $\varphi$ has finite order provided $g \geq 2$.

Corollary 3.3 Let $X$ be a complete smooth curve of genus $g \geq 3$. Suppose that $X$ is trigonal. Namely, there exists a degree 3 morphism $f: X \rightarrow \mathbf{P}^{1}$. Let $\varphi$ be a nontrivial automorphism. Then the number of points left fixed by $\varphi$ is less than or equal to $g+2$.

Proof. As remarked above, $\varphi$ has finite order. If the quotient morphism $\pi$ coincides with the trigonal morphism $f$ above, then $d=3$ and $n \leq g+2$. Otherwise, $d \geq 4$ because $X$ is not hyperelliptic.

Q.E.D.

Corollary 3.4 Let $X$ be a complete smooth curve with genus $g \geq 5$. Let $\varphi$ be a nontrivial automorphism of $X$. Suppose that $X$ is not hyperelliptic nor trigonal. Then the number of points left fixed by $\varphi$ is less than or equal to $(2 / 3) g+2$

Proof. Clear.

A further question is the following:

Question. Let $X$ be a projective smooth variety of dimension $m$. Let $\varphi$ be an automorphism of $X$. Is it possible then to find an integer $n$ such that $\varphi$ is trivial provided $\varphi$ fixes more than $n$ points.

\section{References}

[1] Lionel Bayle and Arnaud Beauville, Birational involutions of $\mathbf{P}^{2}$, Math. AG/9907028.

[2] S. Iitaka, Algebraic Geometry, an introduction to birational geometry of algebraic varieties, Springer, New York-Berlin-Heidelberg, 1982.

[3] M. Miyanishi, Singularities of normal affine surfaces containing cylinderlike open sets, J. Algebra 68 (1981), 268-275. 
[4] M. Miyanishi, Open algebraic surfaces, American Mathematical Society.

[5] D.-Q. Zhang, Automorphisms of finite order on rational surfaces, preprint.

Department of Mathematics

Graduate School of Sciences

Osaka University

1-1 Machikaneyama-cho, Toyonaka,

Osaka 569-0043, Japan

e-mail address: miyanisi@math.sci.osaka-u.ac.jp

and

Department of Mathematics

Himeji Institute of Technology

2167 Shosha, Himeji 671-2201, Japan

e-mail address: kayo@sci,himeji-tech.ac.jp 